\title{
Spatio-temporal shaping of a free-electron wave function via coherent light-electron interaction
}

\author{
Giovanni Maria Vanacore ${ }^{1} \cdot$ Ivan $_{\text {Madan }^{2}} \cdot$ Fabrizio Carbone $^{2}$
}

Received: 14 September 2020 / Accepted: 1 December 2020 / Published online: 24 December 2020

(c) The Author(s) 2020

\begin{abstract}
The past decade has witnessed a quantum revolution in the field of computation, communication and materials investigation. A similar revolution is also occurring for free-electron based techniques, where the classical treatment of a free electron as a point particle is being surpassed toward a deeper exploitation of its quantum nature. Adopting familiar concepts from quantum optics, several groups have demonstrated temporal and spatial shaping of a free-electron wave function, developing theoretical descriptions of light-modulated states, as well as predicting and confirming fascinating phenomena as attosecond self-compression and orbital angular momentum transfer from light to electrons. In this review, we revisit the milestones of this development and the several methods adopted for imprinting a time-varying phase modulation on an electron wave function using properly synthesized ultrafast light fields, making the electron an exquisitely selective probe of out-of-equilibrium phenomena in individual atomic/nanoscale systems. We discuss both longitudinal and transverse phase manipulation of free-electrons, where coherent quantized exchanges of energy, linear momentum and orbital angular momentum mediating the electron-light coupling are key in determining their spatio-temporal redistribution. Spatio-temporal phase shaping of matter waves provides new routes toward image-resolution enhancement, selective probing, dynamic control of materials, new quantum information methods, and exploration of electronic motions and nuclear phenomena. Emerging as a new field, electron wave function shaping allows adopting familiar quantum optics concepts in compositeparticle experiments and paves the way for atomic, ionic and nuclear wave function engineering with perspective applications in atomic interferometry and direct control of nuclear processes.
\end{abstract}

Keywords Electron-light interaction · Coherent phase shaping · Quantum electron manipulation - Wave function engineering - Ultrafast Transmission Electron Microscopy

Giovanni Maria Vanacore and Ivan Madan contributed equally to this work.

Extended author information available on the last page of the article 


\section{Introduction}

Quantum mechanics taught us that matter can be described by multiple wave components whose coherent superposition defines the spatial localization of the associated point-like particle. Typical interference phenomena commonly observed with waves have also been detected for streams of massive particles, such as electrons $[1,2]$, atoms [3], ions [4], and neutrons [5]. Thanks to the modern ability to nano-engineer phase masks with characteristic dimensions of the order of a few nanometers, the spatial control of the coherent superposition of matter waves is becoming a reality. This is commonly referred to as wave function engineering, and it represents a generic concept that finds application in many contexts. For example, in the physics of semiconductor devices and nanostructured materials, wave function engineering refers to the manipulation of the wave function of bound electrons in a solid [6], as also exquisitely demonstrated by the recent examples of twisted bi-layer graphene and similar systems [7]. In this review, we will focus instead on the manipulation of the wave function of elementary particles while propagating in free space. This peculiar endeavor gained interest recently upon the cross-fertilization between the fields of quantum optics and electron microscopy, and can represent an exciting approach able to revolutionize how materials are investigated in electron microscopy. With light waves, several tools have been developed in recent years to shape it into new wave structures characterized by non-trivial properties. Beyond traditional lenses, spatial light modulators and metamaterials have revolutionized optics by enabling efficient control of electromagnetic fields in new ways, leading to significant impact in both fundamental and applied science. For instance, photons that have been engineered to have a well-defined longitudinal and transverse phase profile, can have interesting properties for quantum telecommunications [8, 9], quantum cryptography [10], bio-sensing and imaging with enhanced resolution [11-13].

A similar revolution is now occurring also with matter waves, especially electrons, when similar concepts are being applied to them. Recently, nanofabricated phase masks and electrostatic displays were used to efficiently imprint a vortex modulation on a free-electron wave function [14-16], which could lead to exciting advances in electron microscopy. In parallel, several groups have employed a new paradigm for wave function engineering based on the electron coherent interaction with timevarying electromagnetic fields [17-20]. Such new approach, which has the potential to open new exciting routes for generating radiation and for the dynamical investigation of matter with unprecedented sensitivities, will be the focus of this review.

The method of light-mediated electron beam shaping is enabled by the enhanced interaction between electrons and light in vacuum when mediated by a nanostructure. Such effect is at the basis of the recently developed technique Photon Induced Nearfield Electron Microscopy (PINEM) [21], which demonstrated the ability of transiently probing near-fields at nanoscale in an electron microscope [22-25]. In the following, we will adopt the word PINEM to indicate both the effect and the technique.

In the visible light energy range, the interaction between electrons and photons is very weak and direct photon absorption and emission is forbidden in free-space since simultaneous energy-momentum conservation cannot be fulfilled. This constraint can be overcome if photon momentum is broadened as occurs upon reflection and scat- 
tering or renormalized in dielectrics and polariton modes. In fact, properly designed nanostructures can support so-called surface plasmons polaritons (SPPs) whose near field distribution can reach very high intensities in a very confined space, boosting the cross section for the interaction between the field and a free electron passing by. Differently from the situation in the Kapitza-Dirac effect [26], in which electrons interact with a standing wave made of counterpropagating photons via the ponderomotive force, the interaction mediated by strongly localized SPPs occurs via the linear coupling to electric field. Technically, the electric field associated with the SPP and its temporal and spatial distributions act as a phase mask for the electron wave function, allowing for a light-induced control of both its longitudinal and transverse components. Analytically, the theoretical description of the phenomenon has been given in Refs. [27-29], and builds around the strength of such interaction given by a single complex parameter [20]:

$$
\beta(x, y)=(e / \hbar \omega) \int d z^{\prime} E_{z}\left(x, y, z^{\prime}\right) e^{-i \omega z^{\prime} / v_{e}}
$$

where $\hbar$ is the reduced Planck constant, $\omega$ is the light angular frequency, $E_{z}$ is the electric field component of the SPP along $z$, the direction of propagation of the electrons, and $v_{e}$ is their velocity. Following the interaction with the optical field, the initial electron wave function $\psi_{0}$ gains inelastic components:

$$
\psi_{l}(x, y, t)=\psi_{0} \cdot J_{l}(2|\beta|) \exp \left[\operatorname{ilarg}(-\beta)+i l \omega\left(z / v_{e}-t\right)\right]
$$

where $l$ is the number of exchanged SPP quanta, and $J_{l}(\ldots)$ is the Bessel function of the first kind of $l$ th order, and $t$ indicates the time variable. The interaction parameter is therefore determined by the spatial and temporal distribution of the SPP field, and can thus influence both the longitudinal and transverse components of the electron wave function.

The electrons that underwent this scattering mechanism have information on the field distribution with which they interacted, since it is imprinted in their wave function and thus it can be detected with electron microscopy methods to provide ultrafastresolved movies of local fields in nanostructures [21, 22, 30, 31]. Conversely, when the field distribution is known or designed ad hoc, one can exploit this interaction to impart an arbitrary dynamical modulation on the electron wave function. This can lead to the possibility of coherently controlling via light pulses the quantum state of individual free electrons in the longitudinal direction $[18,20,29,32]$ and in the transverse direction [33]. In the latter case, chiral SPPs were used to imprint a singularity in the phase of the electron wave function resulting in a spiral distribution of its probability current density, thus obtaining an electron beam carrying an orbital angular momentum (vortex beam). Such a phase modulation approach is fundamentally different from monolithic masks technology [14], allowing us to exploit the versatility of dynamical optical control. The phase masks can be switched on and off, or modified with external modulators, and thus the local fields can be modulated dynamically via interference to a scale that can be easily in the attosecond regime, and that has been proposed to be extendable even down to the zeptoseconds [20]. Moreover, such plas- 
monic approach allows for potentially higher throughput of the beam, as attenuation of the beam is not necessary for the creation of phase modulation. These advantages open various intriguing possibilities for future applications, such as the coherent control of the wave function of charged particles of different nature, such as protons, ions and muons, to name a few. In fact, charged particles are easier to manipulate because of their interaction with electromagnetic fields, but for the same reason they can be trapped/absorbed more effectively by material surfaces. For this reason, the manipulation of their wave function via monolithic phase masks is particularly challenging and results in significant losses of the impinging beam intensity and coherence.

In the following, we will review the different methods that have been recently developed for wave function engineering of electrons in free space. In Sect. 2, we will discuss the longitudinal phase modulation and the generation of attosecond electron pulses, while in Sect. 3 we will focus on the transverse phase shaping mediated by linear and angular momentum modulation. Finally, in Sect. 4 we will provide an overview of similar concepts that have been developed in the field of cold atoms and that have interdisciplinary connections with the methods described here. In particular, we will discuss how the manipulation of the wave function of free atoms and ions can be of interest for nuclear physics applications.

\section{Longitudinal electron phase manipulation}

Longitudinal and transverse phase modulation of electron wave function are conceptually and technologically different. The former implies time-modification, while the latter can be achieved employing static or dynamic spatial modulators. From the physical point of view, temporal modification beyond free evolution implies modification of the energy spectrum. By recording electrons spectrum and comparison with theoretical predictions we can access longitudinal phase modulation in a practical setup. From the technological point of view, appropriate time dependent electromagnetic fields should be applied to achieve the desired modulation.

In practice, effects related to the longitudinal phase modulation are observed only upon interaction with visible and near-IR light, whereas in the experiments with $\mathrm{THz}$ radiation or light of longer temporal period, the classical description is sufficient [34]. The simple criterion that discriminates the two regimes is based on the relation between the longitudinal coherence time of an electron wave-packet $\Delta t_{e}$ and the period of the light field oscillation $t_{p}$. For $\Delta t_{e}>t_{p}$ the phase modulation is significant along the wave packet so that PINEM and related coherent effects are observed, while in the opposite case a point particle description is sufficient. This latter regime is appropriate for the description of electron-light interaction in $\mathrm{THz}$ experiments, synchrotron and free-electron laser interactions [34, 35]. The details of the transition between the two regimes have been recently considered theoretically by Pan, Zhang and Gover [36] and have yet to be observed experimentally.

The electron-light interaction in the quantum regime has been considered theoretically by a number of authors. Park, Lin and Zewail [27] have introduced an analytical solution of the time-dependent Schrödinger equation, that provides an elegant relation between the initial and the final wave functions. Employing a number of experimentally 
justified assumptions, they have considered how the moving envelope wave function $\psi$ $(z, t)=g\left(z-v_{e} t, t\right) \psi_{0}(z, t)$ is modified by the light scattered from the nano-object. Here, $\psi_{0}(z, t)=\exp \left[i\left(k_{e} z-\omega_{e} t\right)\right]$ is the carrier wave function, $g\left(z-v_{e} t, t\right)$ is the envelope function with $v_{e}$ the electron group velocity, and $k_{e}$ and $\omega_{e}$ are the electron spatial and temporal frequencies. The PINEM problem is then reduced to finding the time evolution of $g\left(z-v_{e} t, t\right)$, yielding the final state:

$$
g\left(z^{\prime}, t\right)=g\left(z^{\prime}, t_{0}\right) \exp \left[\frac{i e}{\hbar \omega_{p}} \exp \left[-\frac{\left(z^{\prime}+v_{e} \tau\right)^{2}}{4 v_{e}^{2} \sigma_{p}^{2}}\right] \operatorname{Im}\left(\exp \left[\frac{i \omega}{v_{e}} z^{\prime}\right] \beta\left(\frac{\omega}{v_{e}}\right)\right)\right]
$$

where $\sigma_{p}$ is the width of laser Gaussian pulse.

In the limit of continuous illumination, the envelope modulation is given by:

$$
g\left(z^{\prime}, t\right)=g\left(z^{\prime}, t_{0}\right) \exp \left[\frac{i_{e}}{\hbar \omega_{p}} \sin \left[\frac{i \omega}{v_{e}} z^{\prime}+\phi_{0}\right] \beta\left(\frac{\omega}{v_{e}}\right)\right]
$$

This formula provides an intuitive understanding that the laser field sinusoidally modulates electrons phase with modulation amplitude given by the interaction strength $\beta$. We explicitly inserted the phase of the optical carrier $\phi_{0}$ to underline the coherence of the interaction, that will become particularly important in case of electron interaction with multiple pulses.

At approximately same time, Garcia de Abajo, Asenjo-Garcia, and Kociak [28] have developed a theory that provides a relation between the intensities of the different orders of the electron-photon interaction. They have used the Green functions approach, solving the Lipmann-Schwinger equation. As an important result they have predicted the oscillatory behavior as a function of the electric field for the nth order in the limit of long optical pulses and homogeneous electric fields (see Fig. 1b), providing as well the visualization of the occupation probability evolution throughout the interaction.

This prediction have been noticed in early observations in [25, 27], and studied in details by Feist et al. [29] (see Fig. 1c), who have put special effort on creating homogeneous electric field conditions by tightly focusing electron beam and stretching the optical pulses. More importantly, Feist et al. have provided a shift in paradigm by describing the interaction as Rabi-like oscillations on the basis formed not by twolevels, as in atomic Rabi oscillation experiments, but on infinite level quantum ladder Fig. 1a. They reformulated the problem in the second quantization formalism opening up the way for the theoretical studies in a way familiar to quantum optics. In the same publication, they have made the prediction that the free propagation of wave function Eq. (3) would lead to the formation of attosecond trains of electron density at a welldefined distance after the interaction. This prediction has been recently confirmed in several experiments as we describe in details below.

The general applicability limits and "conservation laws" of PINEM theory have been recently formulated by Reinhardt and Kaminer [37]. 

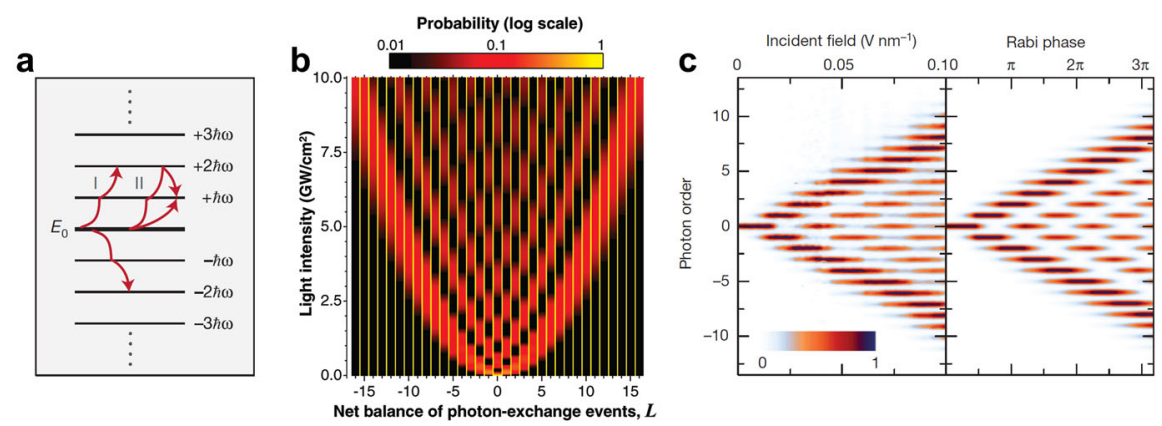

Fig. 1 Longitudinal phase modulation of a free-electron wave function. a The electron-light interaction is described as Rabi-like oscillations induced by the quantum interference between multiple coherent paths in a multi-level quantum ladder. b Theoretical prediction of the oscillatory behavior of the spectral electron occupation probability for the $n$th order $(-16 \leq n \leq 16)$ when computed as a function of the light intensity in the limit of long optical pulses and homogeneous electric fields. $\mathbf{c}$ Experimental verification of the Rabilike oscillation behavior of a single-electron wave packet. A key aspect of the measurement is the creation of a homogeneous electric field condition by tightly focusing the electron beam and stretching the optical pulses. Panels a and $\mathbf{c}$ are adapted from Ref. [29]. Panel b is adapted from Ref. [28]

\subsection{Attosecond pulse trains}

The free propagation of electrons does not change the occupation probability of each energy eigenstate, i.e. the electron spectrum does not depend on the distance after the sample at which it is measured. Yet, each eigenstate of the quantum ladder evolves with a phase that depends on its energy (see Eq. (2)), which implies that the relative phase between the eigenstates change with propagation. As a consequence, the expectation value of the position operator acquires a modulation, with periodicity $v_{e} / \omega$ in the electron reference system, i.e. the probability density acquires the shape of a comb (pulse train).

Priebe et al. have managed to recreate not only the attosecond electron trains, but also to exhaustively characterize them by measuring their Wigner function in the timeenergy domain, which provides an intuitive understanding of the phenomenon [17] (see Fig. 2a, b). The Wigner function is a quasi-probability distribution spanned over the space of two conjugated parameters, analogous to the probability distribution in the phase space in classical mechanics. Since the conjugated parameters cannot be measured simultaneously, it is the integral of the Wigner function along one dimension that has a meaning of a probability distribution in the conjugated dimension. For instance, the integral of the Wigner function along the time dimension provides the probability distribution in energy. Analogously, the Wigner function integrated in energy domain provides the temporal distribution of the probability density.

By comparing the reconstructed Wigner function immediately after the sample with the Wigner function after $5 \mathrm{~mm}$ of propagation, one observes that the quasiprobability distribution has underwent a dispersive reshaping (see Fig. 2b, d): due to a higher phase velocity, the high energy quasi-probability density has advanced in time with respect to the low energy parts. As a consequence, the energy integral of the Wigner function shows regions of increased probability density, spaced by the photon 

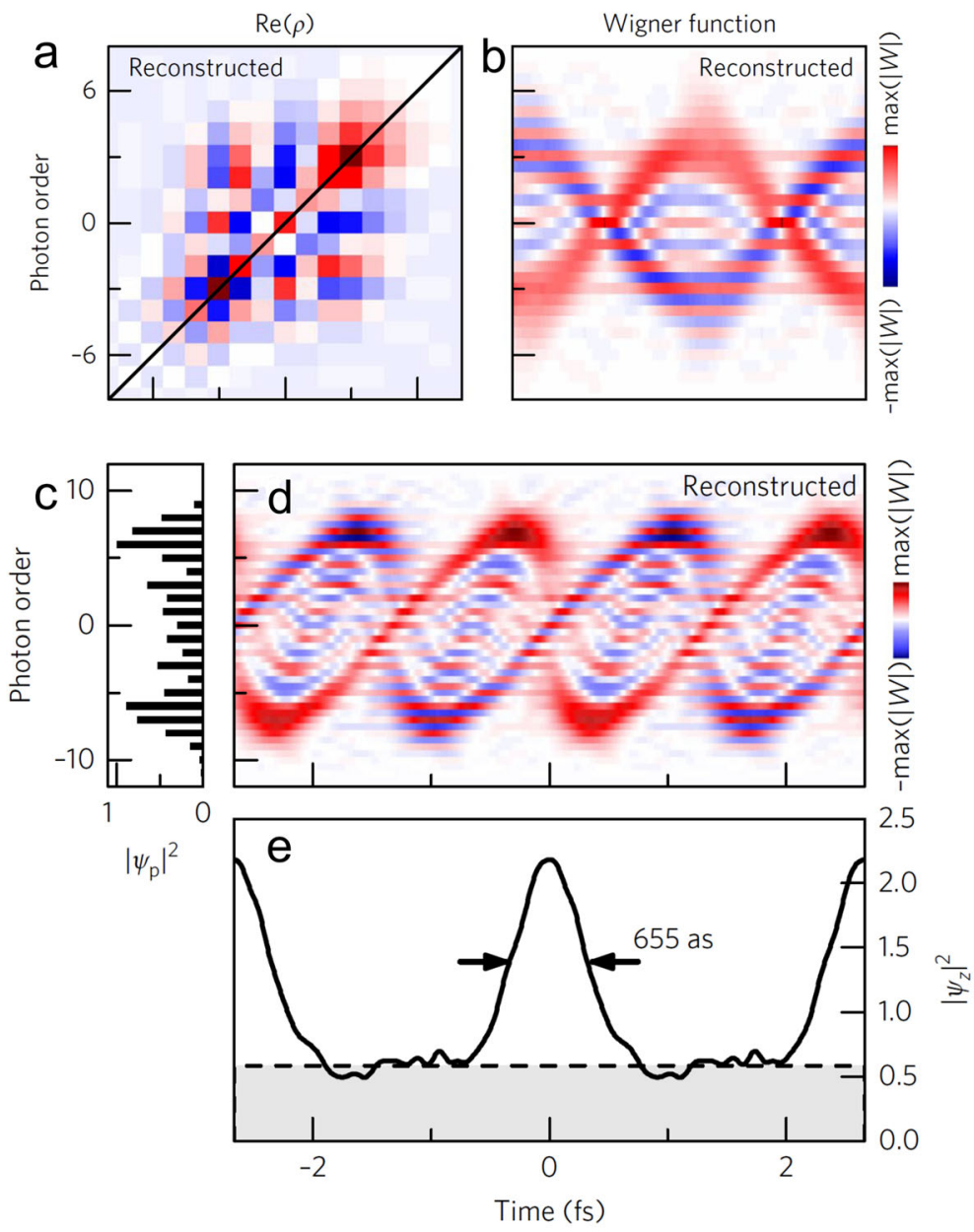

Fig. 2 Generation and characterization of attosecond electron pulse trains. Experimentally reconstructed density matrix (a) and Wigner function (b) of a single-electron wave packet following the interaction with a localized near-field. Here the electron beam is sampled $5 \mu \mathrm{m}$ away (along the z-axis) from the interaction. Experimentally measured spectrum (c) and reconstructed Wigner function (d) of a single-electron wave packet following the interaction with a localized near-field. Here the electron beam is sampled $1.5 \mathrm{~mm}$ away (along the z-axis) from the interaction. One can clearly observes that the quasi-probability distribution has underwent a dispersive reshaping. e The energy integral of the Wigner function shows regions of increased probability density with peak duration of 655 as and spaced by the photon period. All panels are adapted from Ref. [17]

period (see Fig. 2e). One can immediately draw two conclusions about the reshaping. First, there is an optimal distance at which the trains are best defined (the peaks are narrowest). At this distance, a broader energy distribution leads to narrower peaks. Second, there is always non-zero probability density between the peaks.

Priebe et al. have realized the trains with peak duration of 655 as, employing the PINEM interaction with roughly $10 \hbar \omega$ as an energy spread. Given the recent develop- 
ments in strong coupling between electrons and photons extending the energy spread to $100 \mathrm{~s} \hbar \omega$ energy range $[38,39]$, there might be as well significant improvements in the electron density reshaping experiments.

Besides the reconstruction of the Wigner function [17], the attosecond modulation of the electron density has been directly observed in the field-deflection, diffraction and imaging experiments. Naturally, since a single attosecond pulse cannot be in principle extracted due to the associated collapse of the wave function, the current approach allows only the formation of trains, with a duration defined by the electron coherence time. These trains can be used to probe the phenomena that occurs with the periodicity of the train or commensurate to that, most practically the phenomena excited by the same laser source as used for electron phase modulation.

In Refs. [18, 40, 41] a series of pioneering experiments involving an attosecond train of pulses were conducted. The basic observation was the deflection of the electron beam in the laser field with $2.6 \mathrm{fs}$ periodicity. Notably, the deflection is observed also in the diffraction pattern acquired from various crystals, confirming that, at least in simple crystalline semiconductors, there is no observable delay in the diffraction process. This might not be the case in the systems where there is a significant rearrangement of the scattering Coulomb potential due to high-frequency excitations (electrons, plasmons etc.).

The same principle coupled with imaging capabilities allows to demonstrate sub-cycle real-space dynamics of optical fields scattered from the edges of electrontransparent $\mathrm{Si}$ window. This technique provides a direct way to measure the spatial distribution of the phase of the optical fields, and thus it can be useful for light-based nanoscopic applications such as plasmonic light modulators, nanolasers, etc. Importantly, the reported experiments have shown no interaction of the electrons with light in vacuum, which highlights the importance of the momentum conservation and reminds us that the classical description of attosecond pulses in light fields will not be sufficient and momentum consideration as well as the full wave function shall always be taken into account.

The approach discussed here readily provides trains of attosecond pulses. Generation of an isolated attosecond electron pulse remains a milestone to reach. In this direction, there were several theoretical proposals suggesting ways to isolate a single attosecond electron train. Baum and Krausz have proposed a multistage approach where electron pulses would be precompressed to fs duration with a $\mathrm{THz}$ field, such that an optical modulation described above would generate a train with only one pulse [42]. As an alternative, Kozák has proposed to use simultaneous longitudinal and transverse modulation of an electron pulse, that would result not only in temporal train formation but as well in transverse spreading of the train; a single pulse from the train can then be selected with a post-interaction aperture [43]. Another proposal to enable attosecond time-resolved experiments without the constraints of periodicity has been put forward by Hassan et al. [44]. It relies on multi-pulse interaction in a scheme called PINEM-gating described more extensively in the next sub-section.

While the first two approaches rely on dispersive reshaping of the electron density, the third one takes use directly from the attosecond optical pulses. This allows for simpler experimental geometry but reduces the useful flux of electrons. In this respect, theoretically, the first approach allows for nearly no flux reduction, the sec- 
ond approach uses a fraction of electron density that is proportional to the ratio of optical period to electron pulse duration (i.e., around $2.6 \mathrm{fs} / 200 \mathrm{fs}$ for $800 \mathrm{~nm}$ light), while in the third one the flux is proportional to the ratio between the durations of the attosecond optical pulse and the fs electron pulse (e.g., $0.6 \mathrm{fs} / 200 \mathrm{fs}$ ). Such emergence of theoretical proposals allows us to believe that isolated attosecond electron pulses will be demonstrated in the nearest future. A decisive step into this direction has been taken very recently by Morimoto and Baum [45], who have experimentally obtained single-cycle optical control of electrons by generating a modulated electron current with sub-fs structure in time.

Before moving to the next section, we would like to underline here that the PINEM interaction modifies a single electron wave function that preserves coherence until detection. That is to say that, contrary to a naïve view, electrons do not have a welldefined energy after the interaction with the sample but rather remain in a superposition of all energy states. The observed energy upon detection is defined upon wave function collapse at the detector. This aspect is crucial to understand that attosecond trains describe the square of the single electron probability rather than the multi-particle bunches as in FEL or optical attosecond trains. The multi-electron attosecond trains would be impossible due to strong Coulomb repulsion, which is absent when only one electron is concerned.

\subsection{Multi-pulse interaction, Ramsey control, Ramsey-type holography and tomographic reconstruction}

In the discussion above we have already implied that the interaction of the electron with multiple optical pulses is employed for attosecond train observation and characterization. Here, we consider in more details the description of such an interaction and the experimental capabilities it opens up. For clarity reasons and in accordance to current experiments we will only describe electron interaction with two optical pulses.

The experiments in which electrons interact with two optical pulses can be divided in two categories: those in which the coherence between the two pulses is essential, and those in which the description of the coherence can be omitted. The latter case, includes the two pulse experiments either with well separated pulses or pulses without any phase relation, although, as we shall see below, if the two pulses are separated by less than the electron coherence time the coherent effects are still manifested.

In the simplest case, the effect of the first optical pulse is reduced to the creation of a multi-peak energy distribution of electrons limited in time by the optical pulse duration. Since in practical UTEM set-ups the optical pulse duration is several times shorter than the electron pulse these inelastic electrons ${ }^{1}$ generated in the PINEM process have shorter duration than the ZLP background and thus provide the possibility for better time-resolution experiments. This concept was implemented in the pioneering experiments by Hassan and Zewail [46] and is commonly referred to as PINEMgating technique (see Fig. 3d-f). The full capabilities of this technique have been

\footnotetext{
1 We remind the reader that here we ignore coherence effects and thus consider electrons after PINEM interaction as independent particles, much as if there was an instantaneous decoherence imposed on electrons wave function.
} 

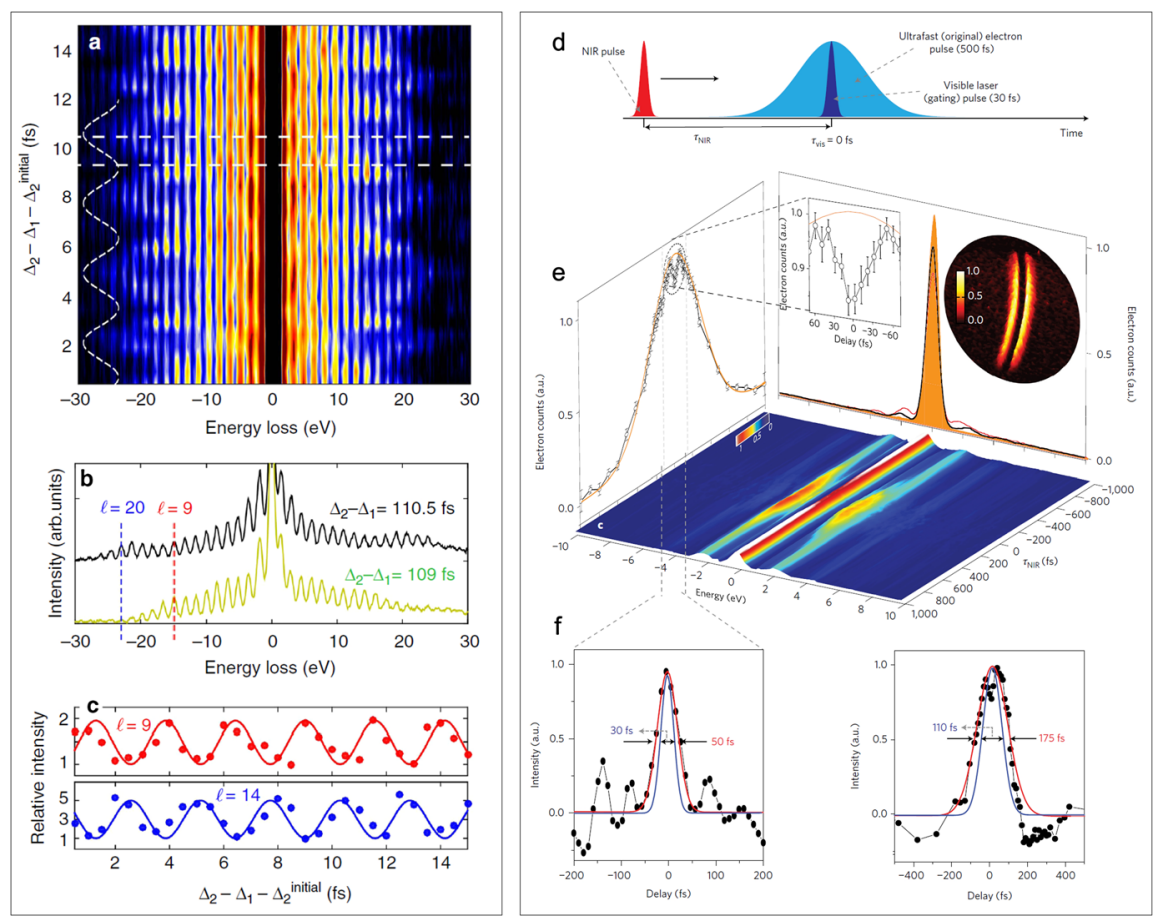

Fig. 3 Time-varying multi-pulse interaction. a, b The electron beam interacts with a semi-infinite temporally modulated optical field distribution produced by a sequence of two mutually phase-locked light pulses impinging on an electron-transparent $\mathrm{Ag}$ mirror. The measured EELS spectra plotted as a function of relative delay between the two optical pulses show clear sidebands molulation. c Relative intensity (full circles) of the $l=9$ and $l=14$ sidebands plotted as a function of time delay between the two optical pulses, exhibiting a periodic modulation of period $\approx 2.6 \mathrm{fs}$ (equal to the optical cycle) and a relative $\pi$ phase shift. Solid curves are least-square fits to the data. d Schematic illustration of the PINEM gating technique for single-pulse time compression. e Time-dependent electron PINEM spectra acquired for the case of a single Ag nanowire when the electron pulse interacts with a gating visible light pulse and a near-infrared pulse used to create the localized near-field. $\mathbf{f}$ The electron distribution inelastically scattered by the NIR-induced near field is temporally modulated by the gating visible pulse and exhibits a temporal duration that depends on the duration of gating pulse. Panels a-c are adapted from Ref. [20]. Panels d-f are adapted from Ref. [44]

further demonstrated with non-degenerate optical pulses by Hassan et al. [44], and it was highlighted that an implementation of this concept with attosecond optical pulses would provide truly isolated attosecond electron pulses, thus removing the limitations of the attosecond train approach. Practical implementation of the PINEM-gating on the attosecond time scale is still to be demonstrated.

PINEM-gating can be used as well to retrieve the optical modification of a material properties with high spatio-temporal resolution. These experiments are conceptually closer to conventional pump-probe experiments in which one pulse is used as a pump that modifies dielectric properties of the material, while the electron pulse locked with the second optical pulse act as a PINEM probe of the time-evolution of the changes of the dielectric susceptibility that enters in Eq. (1) through the $E_{z}$ field. In 
our recent study we have demonstrated the capability to observe nanoscopic details of photo-induced insulator-to-metal transition on a single $\mathrm{VO}_{2}$ nanowire with combined nanometer-femtosecond resolution $\left(10^{-21} \mathrm{~m} \mathrm{~s}\right)$ [47].

Beyond PINEM-gating, additional coherence effects are manifested if optical pulses arrive in the interaction region within the electron coherence time. In a recent publication we have demonstrated that it is possible to coherently manipulate the longitudinal phase of a free-electron wave function using a semi-infinite light field configuration composed of a sequence of two mutually phase-locked light pulses impinging on a mirror and delayed in time by fractions of the optical cycle (see Fig. 3a-c). The profile of the field resulting from such a temporal combination of pulses periodically modulates the energy and momentum of an electron with a period of $2.6 \mathrm{fs}$, providing direct access to the quantum nature of the electron-light coupling.

Practically speaking, the experiment was conducted by employing inverse transition radiation on the surface of the silver film [20]. We observed that two optical pulses separated in time by more than their FWHM were able to significantly alter the PINEM spectrum (Fig. 3a-c). Naively, for short pulses the modulation of the highorder PINEM peaks is thought to be given by the pulse portion that has the strongest electric field, i.e. the center of each pulse. In our set-up, the modification of the peak amplitude due to the presence of the second pulse was negligible. Also, in the temporal region between the two pulses, where interference effects are the strongest, the field amplitude reached only $5 \%$ of the peak amplitude and thus was not affecting the high-order PINEM peaks. The observed coherent oscillations are reproduced by PINEM theory, if one considers an appropriate non-sinusoidal modification of electron envelope. An intuitive understanding of this phenomenon is gained if spectral interference is taken into account. Indeed, two phase locked optical pulses produce periodic modulation of the optical spectrum, with a period defined by the inverse of their temporal separation. Thus, one explains the suppression of the high PINEM orders by the removal of the strongest spectral components from the optical spectrum. This experiment provides an indirect measure of the electron coherence that appears to be of the order of several tens of femtoseconds. That is to be contrasted with the naïve but very common estimate given by the inverse electron spectral width, which in typical photoelectron experiment will be only $\sim 2$ fs. Since there exist no reliable way to measure the electron coherence length in TEM instrument, these experiments provide important steps in the electron wave-packet characterization.

Because such method relies only on the presence of a light-reflecting interface to mediate the electron-light interaction, a particularly appealing consequence consists in the possibility of controlling the electron wave function using photons of different energies. Using high-energy photons all the way to the X-ray regime, our methodology would then allow one to control electrons down to the zeptosecond timescale, as we have theoretically demonstrated using experimental parameters based on the existing technology.

Pioneering experiments exploiting coherence in electron-light interaction have been conducted by the Gottingen group in [32]. They adopted an elegant setup in which to obtain two independent near-fields they have used orthogonal edges of a nanostructure excited with variable polarization laser pulse (see Fig. 4a). In the case of linear polarization diagonal to the structure edges the two near-fields were oscil- 

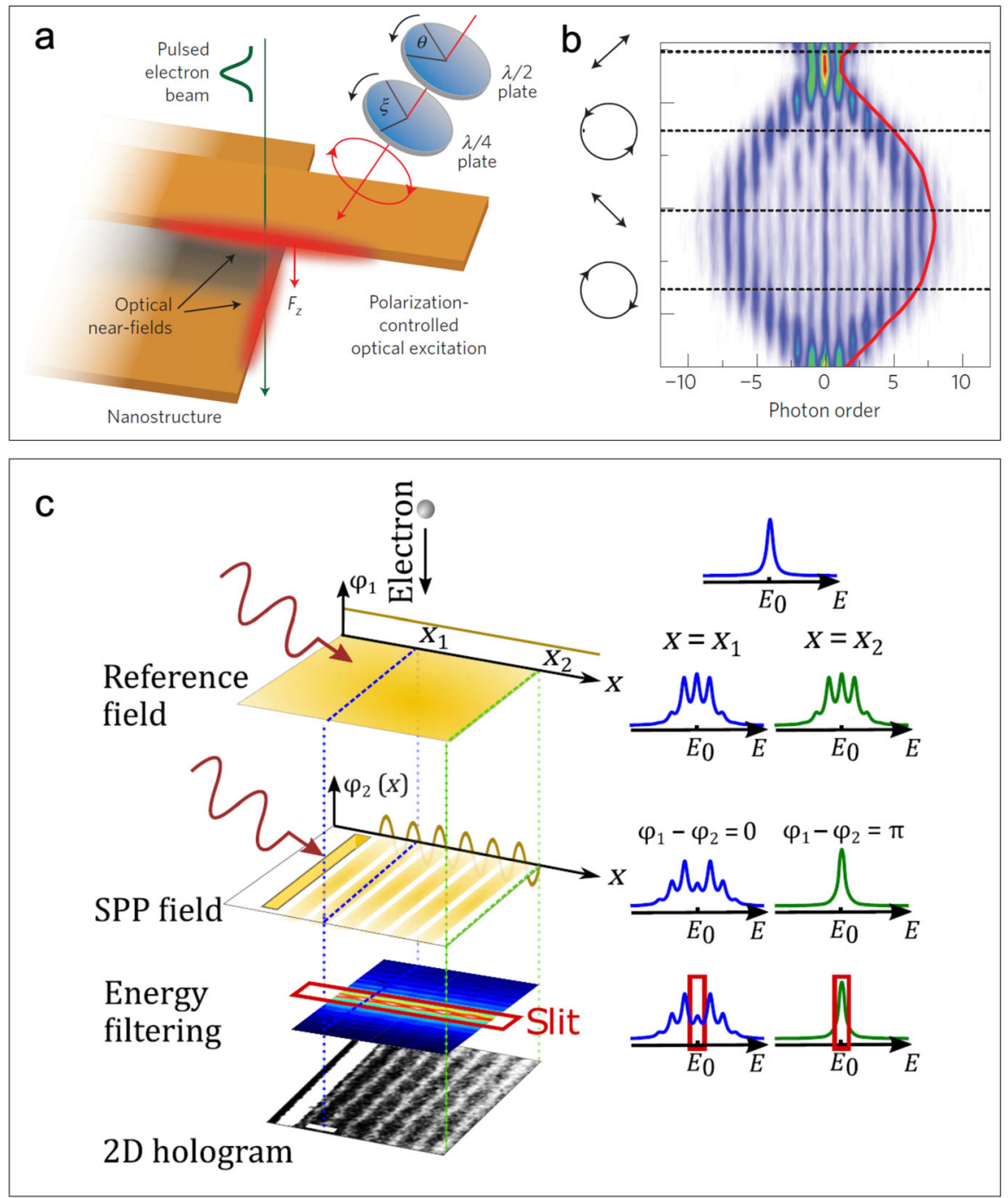

Fig. 4 Ramsey-control and Ramsey-type holographic imaging. a The electron pulse interacts with two spatially-independent near fields created by illuminating orthogonal edges of a nanostructure excited with variable polarization laser pulse. b By gradually varying the ellipticity of the incident field, it is possible to fully map the dependence of the electron spectrum on the relative phase of the near-fields. A strong energy modulation is visible for in-phase oscillations of the fields at the two edges, while there is an almost complete recovery of monochromatic electron spectrum when the two near-fields oscillate in antiphase. Panels $\mathbf{a}$ and $\mathbf{b}$ are adapted from Ref. [32]. $\mathbf{c}$ Experimental implementation of a spatially-resolved Ramseytype experiment for holographic spatiotemporal imaging of propagating surface plasmon polaritons. A homogeneous optical field with a plane phase distribution is used as a reference field. Such field can be then modulated by the interference with near field associated with surface plasmon polaritons excited from a nanofabricated antenna by a separate light pulse. The phase modulation induced by the SPP on the reference field will thus be imprinted on the transverse distribution of the electron wave function that coherently interacts with the two fields. The result is a time-resolved phase-sensitive imaging method. Panel c is adapted from Ref. [31] 
lating in phase while for circularly polarized light the phase shift was $\pm \pi / 4$. By gradually varying the ellipticity of the incident field, the authors were able to fully map the dependence of the electron spectrum on the relative phase of the near-fields (Fig. 4b). This solution is instrumental for a clean experiment as the spatial separation between the two near fields has to be minimal to avoid dispersive reshaping effects, and at the same time the fields should be separated in space so that the effect is not reduced to a simple optical interference.

The description of a double pulse interaction can be done similarly to Eq. (3), in which one uses the final wave function after first interaction as an initial wave function for the second interaction. Since the wave function modulation is a phase modulation of the complex envelope function two consequent interaction would be represented by two exponential phase factors and the total effect can be obtained through the sum of their arguments. In other words one can see the two pulse interaction as a coherent sum of two PINEM fields as if two optical fields were interfering in real space. ${ }^{2}$ The most evident demonstration of such effect is the almost complete recovery of monochromatic electron spectrum in case when two near-fields oscillate in antiphase (Fig. 4b).

The Rabi-description of the PINEM interaction directly allows to interpret this coherent manipulation as a Ramsey-like phase control experiments [32], with an important distinction that one operates with the infinite quantum ladder. In practical terms, there are two implemented applications of the coherent two-pulse interactions: the density matrix reconstruction, and the optical field holography.

In the Ramsey-like experiments, the shape of the modulation of electron wave function due to the first interaction was considered known and given by Eq. (4). Yet, one shall not necessarily limit oneself to this situation and instead one can consider an electron described by a generic density matrix. This might be particularly appropriate if there is an entanglement between the electron and the optical field or a certain degree of decoherence has occurred between two interactions. The density matrix after the first interaction can be built on the quantum ladder states, since this is the experimental basis. The interaction with the second field can be described as a unitary operator that can be changed by changing the relative phase between the two fields. This modifies the density matrix yielding:

$$
\rho_{\text {out }}(\theta)=U(\theta) \rho_{\text {in }} U^{\dagger}(\theta)
$$

where $U(\theta)$ is defined by:

$$
\langle N|U(\theta)| M\rangle=e^{i(N-M) \theta} J_{N-M}(2|\beta|)
$$

The experimental spectrum provides the trace of the density matrix, and as we have seen before it depends on the phase of $U(\theta)$. By acquiring L independent measurements for L different known operators $U(\theta)$, one can fully reconstruct the L-dimensional density matrix, employing the algorithm named SQUIRRELS (spectral quantum interference for the regularized reconstruction of free-electron states) [17]. By obtaining

\footnotetext{
${ }^{2}$ An additional phase factor due to the finite difference in optical paths of two pulses should be taken into account.
} 
the density matrix one can readily judge about the purity of the state as well as build the Wigner function to examine possible density reshaping. In the original paper, the authors have shown the efficiency of the procedure by reconstructing the density matrix of the PINEM state created with $2 \omega$ photons, they also successfully built the Wigner functions of the states after $5 \mu \mathrm{m}$ and $1.5 \mathrm{~mm}$ of free space propagation, to prove the formation of attosecond trains.

The phase sensitivity of the Ramsey-like experiment can be used to study the spatial distribution of the degenerate optical fields in the materials, such as polaritons, guided modes or dipolar excitations (e.g., excitons, plasmons, phonons, etc.). This technique was first implemented by us [31] to study the propagation of a surface plasmon polaritons at the interface between a metal and a dielectric, which behaves as a plasmonic waveguide (see Fig. 4c). The key aspect of this technique is to create an optical field that has a homogeneous in plane phase distribution, which serves as a reference field in conventional holography terms. In the case of thin films or crystals this is easy to achieve, since the laser beam illuminating the surface interacts with electron beam through the inverse transition radiation [20]. In our original study, this modulated beam was then interacting with the plasmon polariton excited from a nanofabricated antenna by a separate pulse. The independent excitation of the two fields can be achieved by properly adjusting the polarization and orientation of the antenna, so that the first pulse does not couple to the SPP and the free-space part of the second pulse does not have polarization component along the electrons trajectory. The signal field of the SPP will be spatially inhomogeneous, and defined by its phase velocity. Provided a large enough field of view and short excitation pulses one also can image the full wave-packet and thus access the group velocity of the mode.

Multiple modes with different phase velocities can be observed simultaneously as we have also demonstrated in Ref. [48]. In a more generalized form, if the electron spectrum is acquired at every position, the amplitude and phase can be completely decoupled and the full field reconstruction can be achieved, as described above. With a careful engineering of the geometry of the sample one would be able to access the complete temporal and (possibly) spatial dispersion, as was exemplified in Ref. [31].

\subsection{Strong coupling regime and cavity QED with electrons}

The above presented description of electron-light interaction was semi-classical in the sense that the laser field was described by an unquantized vector potential. This is generally valid due to the weak coupling between electron and near-fields, being in the range $10^{-4}-10^{-2}$ [49] depending on field localization. That is equivalent to say that at least $10^{2}$ photons per mode are required to obtain an energy exchange between the two fields. In this situation the coherent Glauber, i.e. classical, description of light is appropriate.

In recent years, significant theoretical and experimental efforts have been put into realization of strong coupling regime, in which single electron can efficiently interact with one or few photons. From simple understanding of the coupling constant Eq. (1), this can be achieved through the reduction of the mode volume, or the increase of the longitudinal electric field component of the optical field, and the increase of the 

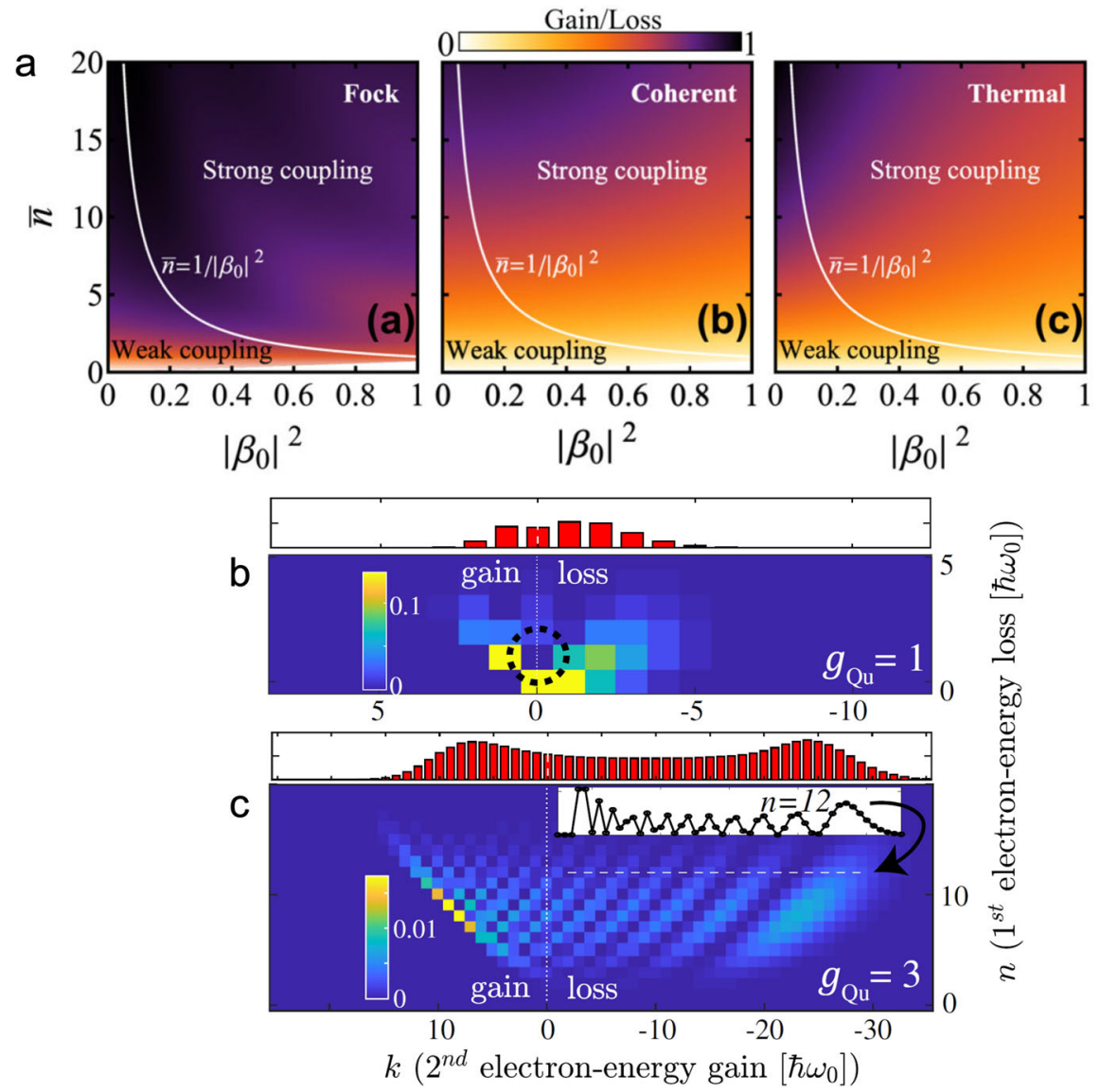

Fig. 5 Electron-light interaction in the strong-coupling regime and quantum light. a Theoretical calculations of the integrated ratio between gain and loss in a PINEM spectrum when the electron wave packet is interacting with Fock (left), coherent (center), and thermal (right) light for both weak-coupling and strongcoupling regimes. Electron spectral distribution theoretically calculated for the case of electron-electron interaction for two distant electrons in a beam mediated by long-lived photons for strong-coupling $g_{Q u}=$ 1 (b) and stronger-coupling $g_{Q u}=3$ (c) regimes. Panel $\mathbf{a}$ is adapted from Ref. [51]. Panels $\mathbf{b}$ and $\mathbf{c}$ are adapted by Ref. [50]

interaction region length (with conservation of velocity matching between electrons and electric field of light). In a pioneering theoretical work [50], Kfir has developed a formalism to describe electron-quantum light interaction, and proposed a practical set-up in which such conditions can be achieved. The system he proposed was a whispering-gallery mode in $100 \mu \mathrm{m}$ long dielectric racetrack cavity, that would realize aforementioned improvements compared to usual near or transient field scenarios. As electron would then efficiently interact with a single photon mode, there would be a clear entanglement between the two and thus correlations between the electron spectrum and number of photons in the cavity would develop (see Fig. 5b, c). Furthermore, since the whispering gallery mode has very weak decay, one can realize the situation 
in which two sufficiently remote electrons, without significant Coulomb repulsion, will interact with the same photon in the cavity. In this case, the two electrons become entangled through the cavity photons, and mutual electron-electron correlations are developed, which can in-principle be experimentally tested in coincidence detection experiments.

Very recently, significant experimental progress has been achieved in this direction. Gottingen and Haifa groups have used spherical [38] and photonic crystal [39] dielectric structures, respectively, to significantly increase the interaction strength. Such effect was manifested in: (i) an increased number of PINEM peaks up to several hundreds, (ii) increased lifetime of the mode reaching picosecond time-scale. Besides that, two remarkable observations were also made. In the whispering gallery system, the chirping of the optical pulse has allowed to quantify the coupling to different modes of the dielectric resonator. Whereas in the photonic crystal case, real-space imaging of the trapped optical field as a function of wavelength and polarization has allowed unprecedented nanoscopic mapping of multiple modes dispersion. These first results provide a solid background for further enhancement of electron-light interaction, which would potentially enable experimental realization of free-electron-photon entanglement.

In his theoretical work, Kfir has limited the discussion to the coherent states, which are naturally excited by free Gaussian electrons. At the same time, the theoretical community is active in the investigation of electron interaction with quantum light. Di Giulio, Kociak and Garcia de Abajo [51] have recently investigated the transition from weak-to-strong coupling for Fock, coherent and thermal light, observing marked differences in the electron spectrum at low photon occupations [51] (see Fig. 5a). One of the particularly elegant results of this work is a shift in paradigm for weak-coupling electron spectrum, in which the ratio between the nth and the first PINEM peaks is directly related to nth order photon correlation function-a common figure of merit in quantum optics experiments.

Earlier, another theoretical work made progress on electron-quantum light interactions [52]. Pan and Gover considered spontaneous and stimulated emission of quantum light by Gaussian and laser modified electron wave-packets. They have concluded that modulation of electron wave function affects the emission of coherent radiation, manifested in modification of the spectral cutoff frequency [53] and appearance of harmonic emission. Instead, no modification in Fock state emission due to electron function modification is predicted. Authors propose clear experimental set-up in which aforementioned effect can be experimentally observed, and ensuing experimental realization have confirmed part of the predictions relating emissions spectral dependence with wave-packet size [54].

\section{Transverse electron phase manipulation}

Besides the longitudinal phase modulation discussed above, several works in the recent years have addressed the transverse manipulation of a free electron wave function. Technologically speaking, transversely manipulating the electron phase and intensity profiles could have strong implications in electron microscopy, providing new routes 
toward image-resolution enhancement, selective probing, low-dose imaging, and depth information. In the following, we will review recent experimental and theoretical efforts, highlighting the challenges, the main scientific contributions, and future trends in the field.

\subsection{Linear momentum manipulation}

Manipulating the transverse linear momentum distribution of a free-electron wave function is the simplest and most straightforward method for electron beam shaping, and can be interestingly adopted for selective probing of specific-symmetry excitation. This has been proved by an elegant experiment from Guzzinati et al. [55], where the authors used a two-lobed beam-Hermite-Gaussian wave-created using a magnetic needle, to distinguish between dipolar and quadrupolar plasmomic modes in Aluminum nanorods.

Coherent and arbitrary dynamical shaping of the electron wave properties down to the sub-femtosecond range and below can be only obtained when using femtosecond light pulses. A semi-infinite light field configuration, as created by light impinging on a totally or partially reflecting electron-transparent plate, interacting with a single-electron pulse has been adopted in several recent studies to demonstrate linear momentum transverse modulation of the electron beam via electromagnetic fields [20, $41,56,57]$. In such condition, the broken translational symmetry of the light field mediates energy and momentum conservation during the electron-light interaction, and the geometrical details of the interrupted wave fronts define the direction and the amount of the transverse momentum exchanges.

In two recent works, Baum et al. [34] have used few-cycles $\mathrm{THz}$ light pulses to induce an instantaneous transverse momentum transfer to the electron beam. The resulting deflection was proportional to the integral of the Lorentz force along the electron's trajectory and closely followed the electric field's temporal profile (see Fig. 6a). For such low-frequency pulses, the temporal coherence of the electron wave packet is always smaller than the light period $\left(\Delta t_{e}<t_{p}\right)$, and therefore the electron spatial distribution follows the electric field oscillations (classical framework). In the opposite limit, i.e. for an electron temporal coherence longer than the light period ( $\Delta t_{e}>t_{p}$, such as in the case of visible light illumination), the temporal evolution of the transverse electron momentum profile would mimic that of the vector potential of the electromagnetic field [58]. This can be seen in another recent work from Morimoto and Baum [18], where the authors used a dielectric membrane illuminated by femtoseconds light pulses with central wavelength of $1030 \mathrm{~nm}$, and monitored the time-dependent momentum kick induced on a 70-keV electron wave packet by the resulting semi-infinite light field (see Fig. 6b). Similar results have also been obtained by us [20] using 800 -nm femtoseconds light pulses impinging on a $50-\mathrm{nm}$ silver layer. Here, we have measured the subtle transverse momentum modulation of 200-keV single-electron pulses by mapping their reciprocal space distribution in high-dispersion diffraction mode. The results are shown in Fig. 6c, where a clear streaking of the electron beam appears along the $\mathrm{k}_{\mathrm{X}}$ direction as a result of the net momentum exchange. From a theoretical viewpoint, the physical origin of this behav- 

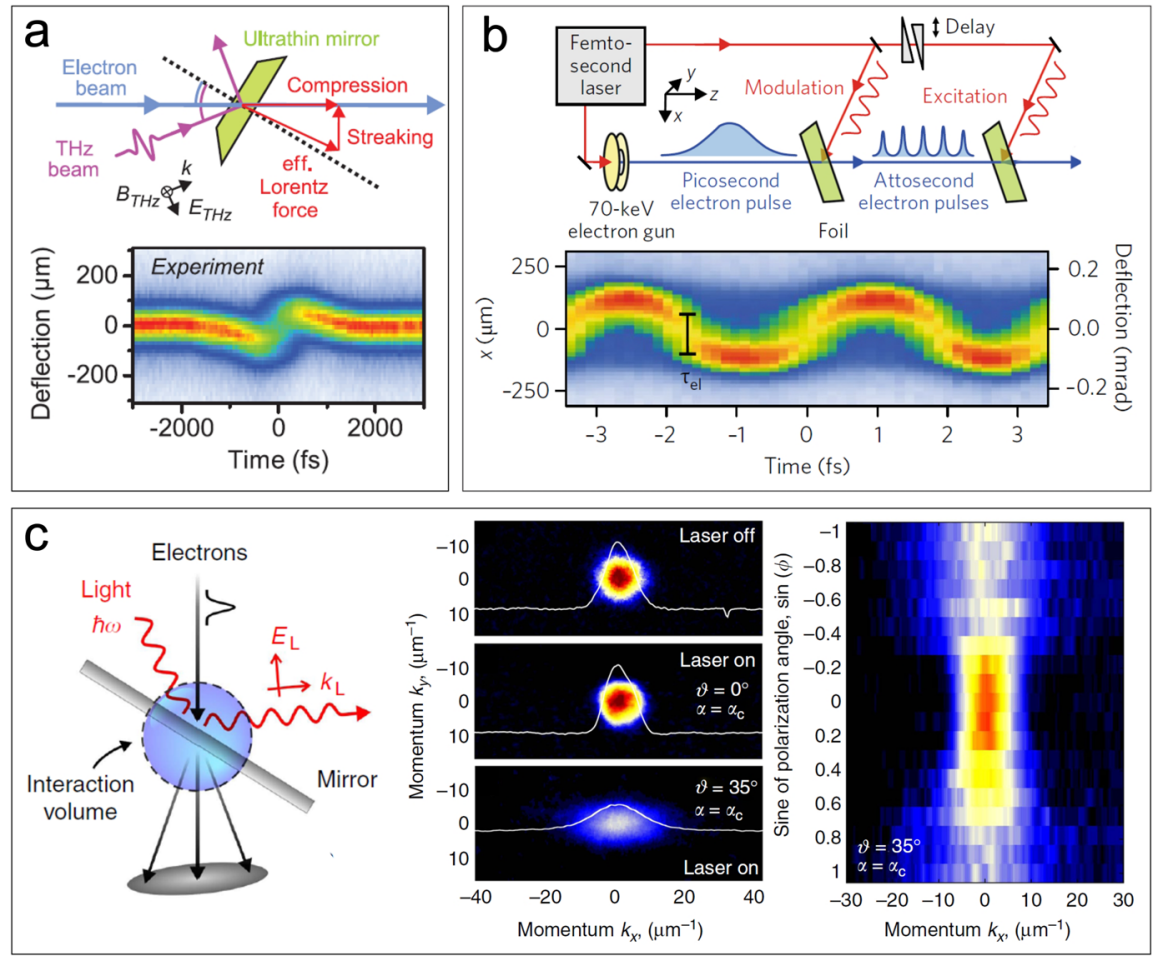

Fig. 6 Transverse momentum modulation of a free-electron wave function by semi-infinite light fields. A light field is impinging on a totally or partially reflecting electron-transparent plate and interact with a singleelectron pulse. a A few-cycles THz light pulse is used to induce an instantaneous transverse momentum transfer to the electron beam. The resulting deflection is proportional to the integral of the Lorentz force along the electron's trajectory and closely follows the electric field's temporal profile. Adapted from Ref. [34]. b A dielectric membrane is illuminated by femtoseconds light pulses with central wavelength of $1030 \mathrm{~nm}$, and the time-dependent momentum kick induced on a 70-keV electron wave packet is monitored as a function of time. In this case of electron temporal coherence longer than the light period, the temporal evolution of the transverse electron momentum profile mimics that of the vector potential of the electromagnetic field. Adapted from Ref. [18]. c 800-nm femtoseconds light pulses are impinging on a 50-nm silver layer (left). The transverse momentum modulation of 200-keV single-electron pulses is elucidated by mapping their reciprocal space distribution in high-dispersion diffraction mode. A clear streaking of the electron beam appears along the $\mathrm{k}_{\mathrm{X}}$ direction as a result of the net momentum exchange between electrons and photons (center). The electron-beam momentum spread is also dependent on the rotating polarization of the light wave (right). Adapted from Ref. [20]

ior is captured by a single complex coupling coefficient that defines the electron-light interaction strength (Eq. (1)), which strictly depends on the electric field component along the electron's propagation trajectory. This was also experimentally verified by monitoring the changes of the electron-beam momentum spread as a function of the rotating polarization of the light wave (see right panel in Fig. 6c).

It is important to note, that in our experiment [20] the transverse spatial coherence of the electrons was made almost comparable with the light wavelength. This is an extremely important feature, since it implies that a coherent quantized interaction between the electron wave function and the light wave has to be expected, rather 


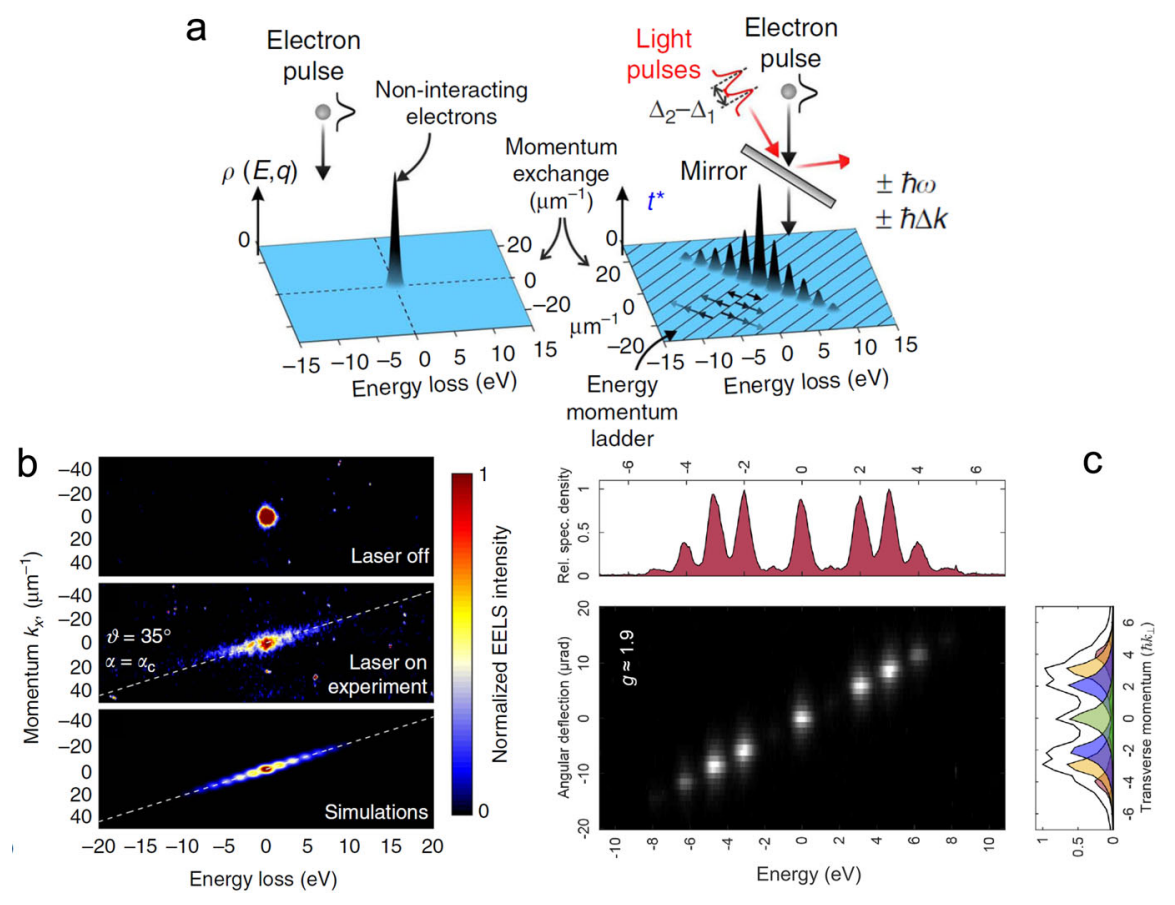

Fig. 7 Energy-momentum mapping of the quantized electron-light interaction. a When the transverse spatial coherence of the electrons is comparable with the light wavelength, a coherent quantized exchange of energy and momentum between an electron wave function and a light beam has to be expected. $\mathbf{b}$ and $\mathbf{c}$ Experimental demonstration of the quantized nature of the electron-light coupling obtained by monitoring the electron intensity distribution within an energy-momentum space. Panels $\mathbf{a}$ and $\mathbf{b}$ are adapted from Ref. [20], while c is adapted from Ref. [57]

than a purely classical deflection as mediated by the Lorentz force, which is generally negligible under excitation at optical photon frequencies [20]. We were thus able to observe for the first time the simultaneous quantized exchange of energy and momentum between an electron wave function and a light beam, providing a direct measure of the degree of such interaction. This observation, which attested the primary role of the quantum nature of the electron-light coupling, was demonstrated by monitoring energy-momentum maps, simultaneously accessing inelastic energy exchanges and transverse-momentum exchanges (see Fig. 7a, b). The streaking of the electron beam occurred along a line in energy-momentum space with slope given by $\mathrm{q}_{\mathrm{T}, \mathrm{x}} / \hbar \omega$, where $\mathrm{q}_{\mathrm{T}, \mathrm{x}}$ is the transverse component of the transferred momentum along $\mathrm{x}$. In the limit of small light-incidence angle, it admits the expression:

$$
q_{T, x}, \approx(\omega / c) \cos \vartheta \sin \vartheta
$$

where $\vartheta$ is the membrane tilting angle with respect to the electron's propagation direction $\mathrm{n}$, which is quasi-collinear with the incident laser wave vector. For every photon absorption/emission event, the electron gains/loses a quantum of energy $\hbar \omega$ and a quantum of momentum $\hbar \mathrm{q}_{\mathrm{T}, \mathrm{x}}$ along $\mathrm{x}$. Such experiment yields a direct observation 
of the simultaneous quantized exchange of energy and transverse momentum between a propagating light wave and a free-electron, and shows the unique ability of our technique to map transient energy exchanges in momentum space. Very recently, Feist et al. [57] using a similar semi-infinite light field configuration, showed that by further increasing the lateral coherence of the electron beam well above the optical wavelength, it is indeed possible to obtain high-purity free-electron energy-momentum states (see Fig. 7c). These results demonstrated that a free-electron wave function can be shaped as a coherent superposition of quantum ladder states, each characterized by a welldefined energy and linear momentum, by the imprinted longitudinal and transverse phase modulation.

In the experiments above, we have discussed the interaction of the electron wave packet with a single propagating light wave, which resulted in a series of peaks along a single direction in the energy-momentum space as defined by Eq. (7). Very interesting is the case when electron-light coupling is mediated by two counter propagating light waves with opposite momenta. When occurring in free-space, this is the so called Kapitza-Dirac (KD) effect, which is related to the diffraction of electrons scattered from the light grating as a result of the action of the ponderomotive force of the light field [26, 59]. Garcia de Abajo et al. [60] have recently discussed the case where the two counter-propagating light fields are actually represented by two surface plasmon polaritons sustained by a metal surface or metal/dielectric interface (see Fig. 8a). In such configuration, which basically considers a plasmonic Fabry-Perot cavity, electrons are shown to undergo strong diffraction in both elastic and inelastic components of their wave function, resulting in a 'butterfly'-like pattern in the energy-momentum space. The effect is the plasmonic equivalent of the KD effect, although here the interaction is no longer mediated by the ponderomotive force as in the case of freespace light waves but rather from the electric field associated with the surface plasmon polaritons. In particular, the plasmonic field would both locally modify the phase of the electron wave function through multiple inelastic exchanges, and the amplitude of the electron wave function by transferring large intensity from elastic to inelastic channels at transversal positions corresponding to maximum plasmonic field strength. A similar KD-configuration was also studied, both analytically and numerically, by Talebi and Lienau [61]. In particular, they considered the interaction of a low-energy electron pulse with a standing wave of light formed by two counter-propagating laser fields in free space with a well-defined inclination angle between each other. For sufficiently high light intensities and at specific electron velocities, they predicted the presence of a variety of additional features in the electron momentum distribution beyond the elastic diffraction as expected in the normal KD effect (see Fig. 8b). The authors attributed such complex patterns to a quantum path interference between electrons scattered by the ponderomotive force of the standing light wave and electrons undergoing direct light absorption or stimulated emission processes.

The works discussed here are extremely promising and could prompt the development of new microscopy methods in which the limitation imposed by EELS energy resolution is lifted for large momentum transfers, such as in the dynamic imaging of low-energy phonons. Furthermore, they clearly demonstrate the ability of external electromagnetic fields to modulate the electron phase and intensity distributions, pro- 

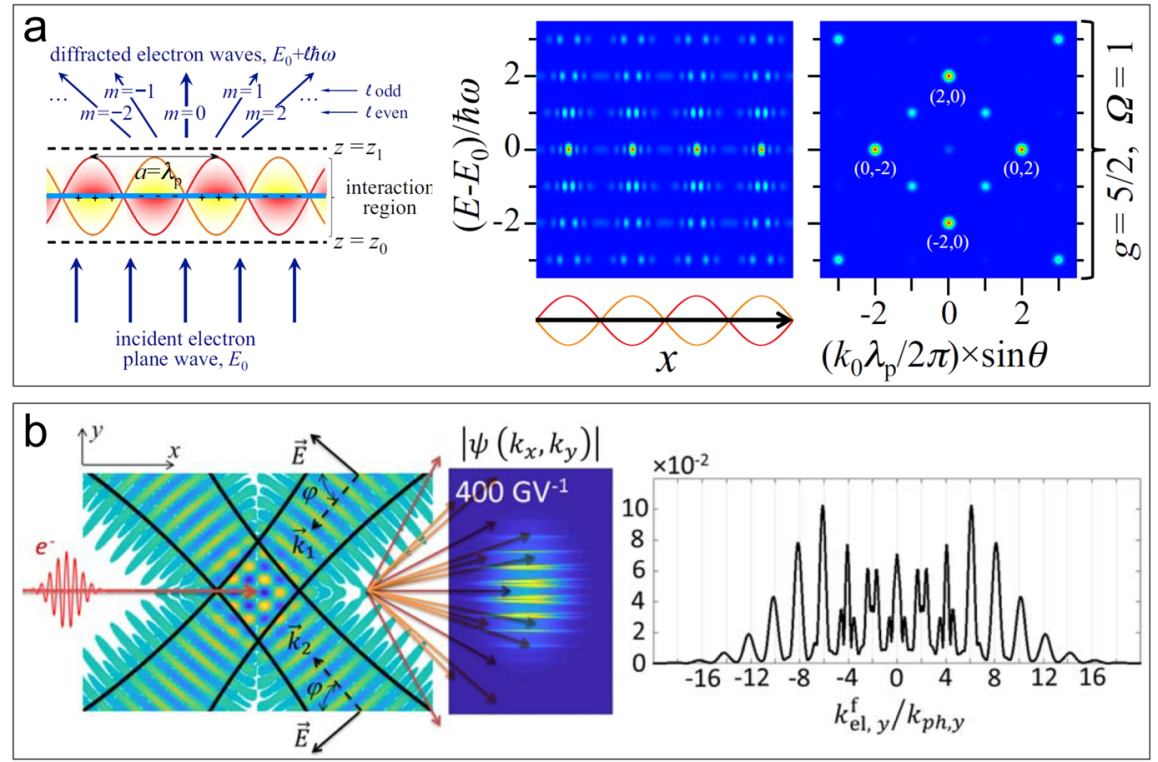

Fig. 8 Transverse momentum modulation of an electron wave packet by multiple light beams. a Plamonic Kapitza-Dirac Electrons scatter from the light field configuration created by two counter-propagating surface plasmon polaritons sustained by a metal/dielectric interface. Electrons are shown to undergo strong diffraction in both elastic and inelastic components of their wave function, resulting in a 'butterfly'-like pattern in the energy-momentum space. Adapted from Ref. [60]. b Two-dimensional Kapitza-Dirac A low-energy electron pulse is interacting with a standing wave of light formed by two counter-propagating laser fields in free space with a well-defined inclination angle between each other. For high light intensities electrons scattered by the ponderomotive force of the standing light wave interfere with electrons undergoing direct light absorption or stimulated emission processes, creating a complex momentum distribution. Adapted from Ref. [61]

viding coherent and arbitrary dynamical shaping of the electron wave properties down to the sub-femtosecond range and below.

\subsection{Imprinting and dynamically modulating orbital angular momentum}

An extremely interesting application of transverse electron phase manipulation is the ability to create electron beams carrying a well-defined orbital angular momentum (OAM), and thus with a specific chiral character. This is very appealing for probing collective and quasi-particle excitations exhibiting a chiral order, which are of significant interest for both applied and fundamental science. Chiral order has been found to play a crucial role in controlling the behavior of quantum materials, whose physics is governed by the interplay between reduced dimensionality, topological protection and strong correlations. Despite recent advances, investigation of chirality in condensed matter, specifically in low-dimensional quantum systems, is still at its infancy. So far, information is mainly obtained using optical probes [62], which however exhibit an inherently low sensitivity in the case of nanoscale structures and a poor spatial resolution because of the long wavelength of light. 
High energy electrons have a larger cross-section of interaction with matter than light, they have a much smaller wavelength — picometer range—, and can be focused to a spot size down to the nanometer range or below. Electrons become sensitive to chiral structures when carrying a well-defined orbital OAM, which can be obtained by imparting a vortex structure on their transverse phase profile $[14,16]$. Vortices are stagnant points at which the phase is undefined, while along a contour around them it varies by an integer multiple $m$ of $2 \pi$. The magnitude and sign of the quantization number $m$, also known as the topological charge, indicate the amount and the handedness, respectively, of the phase cycles surrounding the vortex. The presence of such singularity is accounted for in the expression of the electron wave function by a phase factor $\exp (i m \phi)$, where $\phi$ is the azimuthal angle. A vortex electron wave is thus an eigenstate of the OAM operator with eigenvalue $m \hbar$, where $\hbar$ is the reduced Planck constant. The electron OAM thus plays the role of the light spin angular momentum (circular polarization), and angular momentum conservation in elastic and inelastic scattering of vortex electrons from chiral structures will thus result in a different cross-section according to their relative helicity. Namely, the degree to which vortex electrons are scattered will thus depend on the handedness of their helical wavefronts. The main advantages of using vortex electrons with respect to light are that for electrons many selection rules do not apply and, while the light spin can be only $\pm \hbar$, the electron OAM assumes arbitrarily large values $(m \hbar$, with $m= \pm 1, \pm 2, \pm 3, \ldots)$. All these aspects point to a strongly enhanced chiral interaction between a vortex electron beam and a chiral material, as predicted in numerous situations involving magnetic materials [16], plasmonic nanostructures [63], crystalline strains [64], and macromolecules [63].

Electron beams that carry OAM can be produced by means of passive devices that directly modify their wave structure, such as spiral phase plates [65], amplitude holograms [66] and phase holograms [67, 68]. The electron's charge also allows a vortex to be obtained by other methods, such as magnetic needles [69], programmable electrostatic displays [70] and tunable electrostatic phase devices [71].

So far current schemes have relied on passive, static or slowly-varying (millisecond or longer) modulation of the electron wave function, restricting the range of possible applications mainly to the investigation of ground-state processes. However, a huge amount of information resides in the exploration of the non-equilibrium dynamics. The ability to visualize the transient behavior of chiral orders in nanoscale quantum materials is, in fact, crucial for controlling topological states, for manipulating electronic phase transitions and for modulating carrier transport. This is only possible when dynamically investigating such systems with appropriate spatial, temporal, energetic and momentum resolutions, while simultaneously monitoring their coupling to other degrees of freedom (lattice, spin, topology). An alternative approach, which would be able to address these issues, would rely on the discrete exchange of OAM between interacting relativistic electrons and optical electromagnetic waves. Barwick et al. [72] have proposed an all-optical method for creating electron vortices using the Kapitza-Dirac effect (see Fig. 9b), where two counter-propagating vortex light beams would be adopted to transfer OAM to an interacting electron wave packet. More recently, Cai et al. [73] have theoretically calculated the cross-section for OAM transfer between electrons and localized chiral plasmons (see Fig. 9a), and determined that large amounts of OAM exchanges can be obtained by exploiting multi-photon 

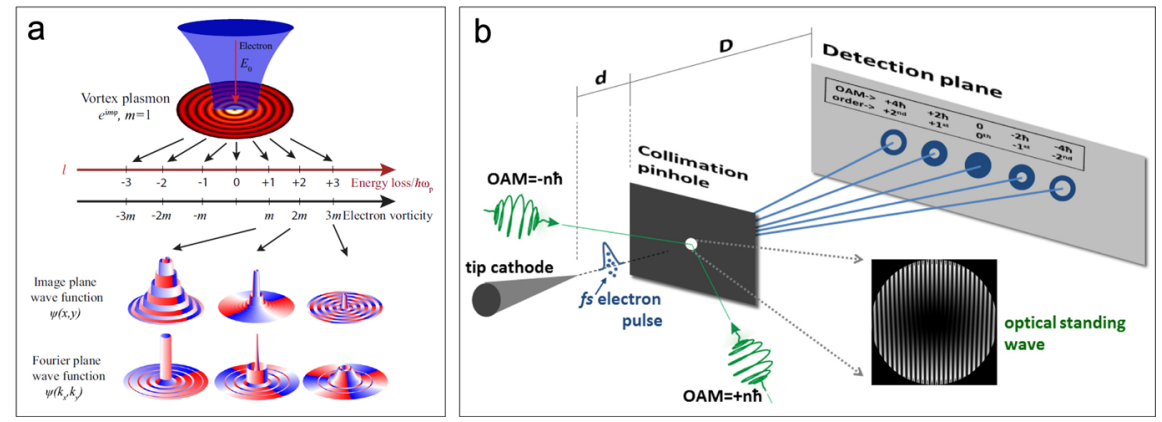

Fig. 9 Proposed theoretical approaches for electron-light orbital angular momentum (OAM) transfer. a The interaction between a free-electron and localized chiral plasmons is predicted to drive large amounts of OAM exchanges from the plasmons to the electron by exploiting multi-photon processes. Adapted from [73]. b The interaction of a free-electron with the optical grating created by two counter-propagating vortex light beams is predicted to form electron vortices in the diffraction beams. Adapted from Ref. [72]

processes. In a recent work [33] we have experimentally demonstrated that ultrafast vorticity can be imparted on a single electron wave packet by interaction with an optically excited femtosecond chiral plasmonic near field. Compared with static approaches using passive phase masks, this method offers a higher degree of scalability to small length scales and a highly efficient dynamic phase control. In fact, thanks to the ability to rapidly manipulate the driving ultrafast plasmonic field, such method allows to coherently control the properties of an electron vortex beam with sub-fs precision.

In particular, we have generated an ultrafast vortex electron beam by means of a spatially-confined optical field carrying OAM as created by the excitation of chiral surface plasmon polaritons sustained by a metal/dielectric interface (see Fig. 10a). The formation of chiral plasmons [74] relies on the spin-to-OAM conversion [75] from circularly-polarized light in non-paraxial scattering from a nanoscale cavity and is a clear manifestation of the strong spin-orbit coupling of light when confined to subwavelength scales. The quantized inelastic coupling between a free-electron and such field configuration is thus responsible for an efficient transfer of a nonzero topological charge from the chiral SPP to the transverse profile of the electron wave function, which now assumes a helical phase distribution. For a chiral plasmon polariton, the interaction strength $\beta$ is such that $\arg (-\beta)= \pm n \phi$, where $n$ defines the plasmon azimuthal order while the sign depends on the relative handedness. By plugging such expression in Eq. (2), this linear dependence translates into a corkscrew phase factor in the scattered electron wave function:

$$
\psi_{l} \propto \psi_{0} \exp ( \pm i \ln \phi)
$$

which therefore carries an OAM with eigenvalues given by $\pm \ln \hbar$. This was verified by monitoring the far-field images of the electron wave function in the transverse momentum space: $\Psi_{l}\left(k_{x}, k_{y}\right)=F\left\{\psi_{l}(x, y)\right\}$, where $F\{\ldots\}$ denotes the Fourier transform operator, before and after the interaction with the chiral near field (see Fig. 10b). Here, 

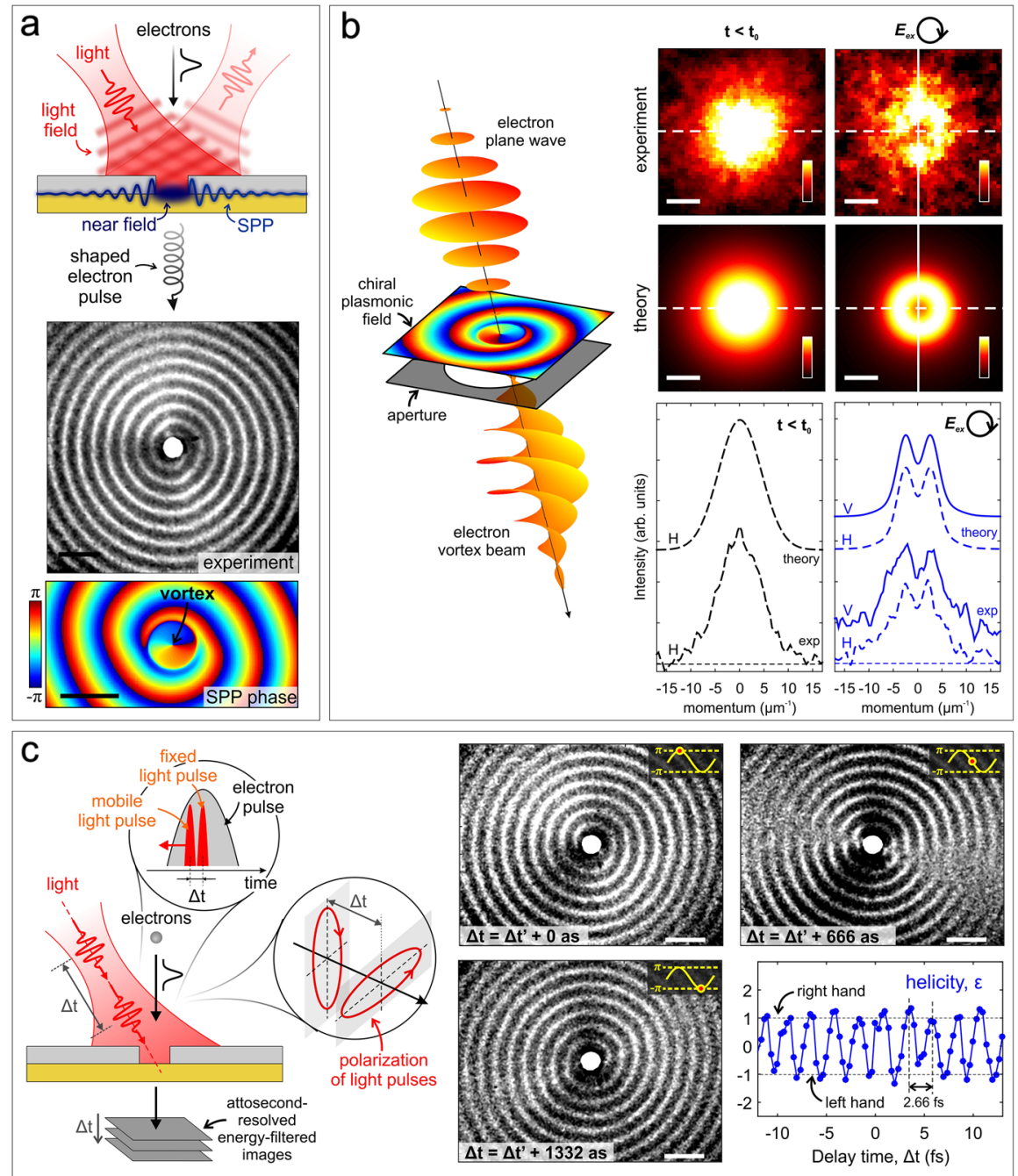

Fig. 10 Ultrafast generation and control of electron vortices by chiral plasmonic near-fields. a A single electron wave packet interacts with an optically excited femtosecond chiral plasmonic near field carrying orbital angular momentum (OAM). The experiments show the real-space phase distribution of the chiral field, which exhibits a characteristic spiral shape with a vortex singularity in the center. b The quantized inelastic coupling between a free-electron and such chiral field is responsible for an efficient transfer of a nonzero topological charge from the chiral SPP to the transverse profile of the electron wave function, which now assumes a helical phase distribution. This was experimentally verified by monitoring the far-field images of the electron wave function in the transverse momentum space in absence and in presence of such chiral field. c Sub-femtosecond dynamic control of electron vorticity. Two phase-locked light pulses are used with a relative delay, $\Delta \mathrm{t}$, varied in steps of 334 as. When the two pulses are set with a cross-polarization, the handedness of the spiral plasmonic phase distribution, and thus the electron vorticity, is switched from clockwise to anticlockwise in about $1.3 \mathrm{fs}$. All panels are adapted from Ref. [33]

the presence of a well-defined OAM is verified by the characteristic 'donut'-like shape of a vortex electron beam. 
An extremely interesting feature of our approach is the ability to dynamically control the vortex beam properties (see Fig. 10c). The vorticity of the electron wave function $\psi_{l}$-being defined by the vorticity of plasmonic field - can be engineered through optical pulse engineering. Therefore, one can produce pulses of alternating vorticity, with an alternation period that matches a magnetic or chiral mode in the sample. The second advantage of such dynamic capabilities is the utilization of rapid vorticity alternation for the studies of irreversible phase transformations or dichroic experiments. In the latter case, the alternation of vorticity has to be synchronized with the detection read-out, allowing for a homodyne detection scheme able to remove background unrelated to dichroism, the major source of noise in dichroic experiments.

Besides the dynamical aspects, the utilization of plasmonic fields brings technological advantages: in principle, plasmonic phase modulation does not require attenuation of electron beam, providing more flux for detection. Finally, active spatial distribution of nanoscopic optical fields is routinely engineered, and multipurpose phase plates with more sophisticated phase profiles can be placed for a broad range of experiments, these can include dipolar/quadrupolar or higher order chiral modulation of electron wave function, for desired enhancement of interaction with the sample.

\subsection{Laser-based phase plates}

The large amount of results discussed above clearly demonstrates the advanced level of sub-fs transverse electron phase manipulation reached nowadays. The next technological step will involve the exploitation of such potential in order to design and realize all-optical rapidly-programmable phase masks for electrons, which would be able to revolutionize the way materials are investigated in electron microscopy. A first theoretical approach toward this objective has been recently provided by Konečná and Garcia de Abajo [76], who conceived a photonic aberration corrector made of an electron-transparent metallic plate where it will be imprinted a well-defined optical near-field externally controlled by a computer-programmable spatial light modulator (see Fig. 11a). The authors theoretically demonstrated that a field configuration composed of a superposition of zero-order Bessel light beams with laterally changing wave-vectors would be able to correct the spherical aberration induced in the electron beam by the TEM electron optics, provided that a Wien filter is used to select only the electrons that have interacted with the electromagnetic field. On the experimental side, in a recent publication Schwartz et al. [77] have implemented a continuous-wave (CW) laser-based phase plate using a Fabry-Perot optical cavity integrated within the electron microscope (see Fig. 11b). They showed that the ponderomotive interaction between the electron beam and such continuous standing light wave creates a welldefined phase contrast in the TEM, which strongly depends on the light intensity and enhances the image contrast at low spatial frequencies. It is essential to note that elastic ponderomotive interaction does not change energy spread of electron beam observed in PINEM and thus avoids chromatic aberrations problematic for high-resolution imaging. Although the use of a CW laser system would certainly remove the limitations associated with the laser duty cycle or synchronization jitter proper of a pulsed laser setup, the adoption of femtosecond light pulses is the only way to dynamically shape 

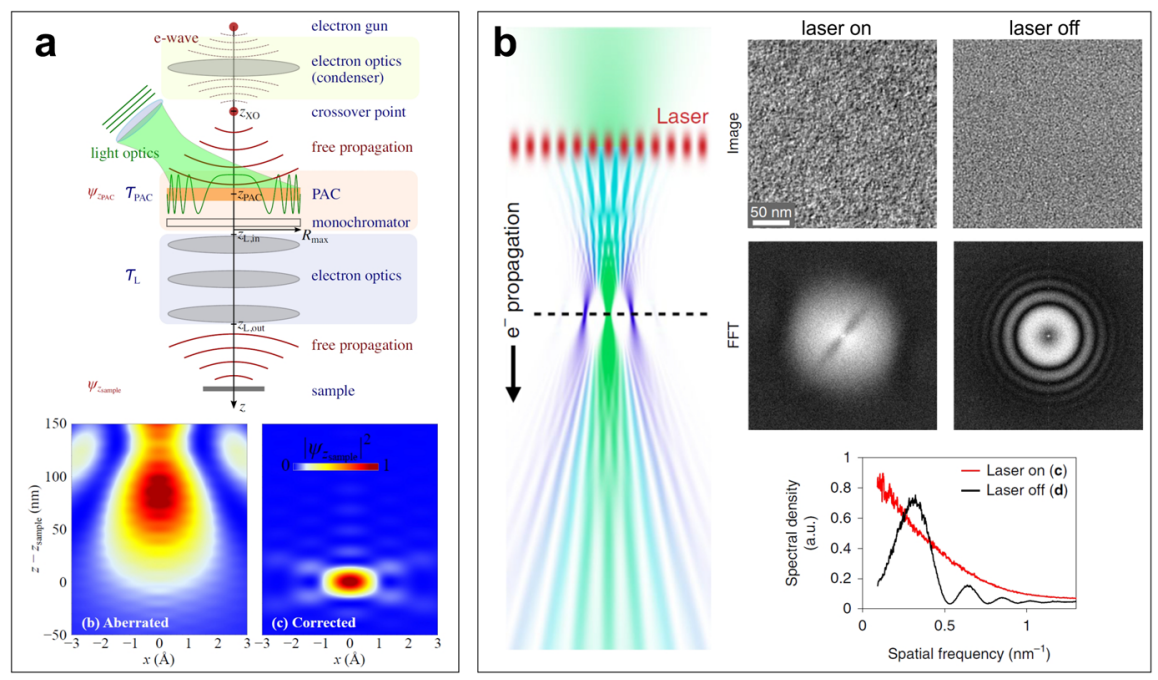

Fig. 11 Laser-based electron phase plates. a Theoretical proposal for a photonic aberration corrector made of an electron-transparent metallic plate where it is imprinted a well-defined optical near-field externally controlled by a computer-programmable spatial light modulator. A superposition of zero-order Bessel light beams with laterally changing wave-vectors would be able to correct the spherical aberration induced in the electron beam. Adapted from Ref. [76]. b Continuous-wave (CW) laser-based phase plate using a Fabry-Perot optical cavity integrated within the electron microscope. The interaction between the electron beam and such continuous standing light wave creates a well-defined phase contrast in the TEM. Adapted from Ref. [77]

the free-electron wave function on the suitable spatio-temporal scales for investigating non-equilibrium dynamics of nanoscale materials.

\section{Towards wave function engineering of composite particles (neutrons, atoms, ions and protons)}

Besides electron waves, the possibility of twisting coherent matter waves has been recently demonstrated also for neutrons and atoms [5, 66, 78]. In particular, imparting orbital angular momentum on fundamental composite particles have been shown to hold promise to manipulate their inner structure [79]. In the case of neutrons, it was recently pointed out that patterning their wave function by imprinting a well-defined OAM could be useful to investigate their inner arrangement of quarks and gluons, which are inherently affected by the external angular momentum [79]. A similar proposal was put forward to study the origin of the proton spin [33].

The vast majority of these experiments are carried out by passing a coherent stream of particles through a passive element - a phase mask - acting on their wave nature to exploit quantum interference effects to modulate their wave function. Such ability to twist matter waves is a very recent achievement in experimental physics whose full potential has yet to be explored. We note that, in particular, manipulating the wave function of composed particles, such as atoms and ions for instance, would mean being 

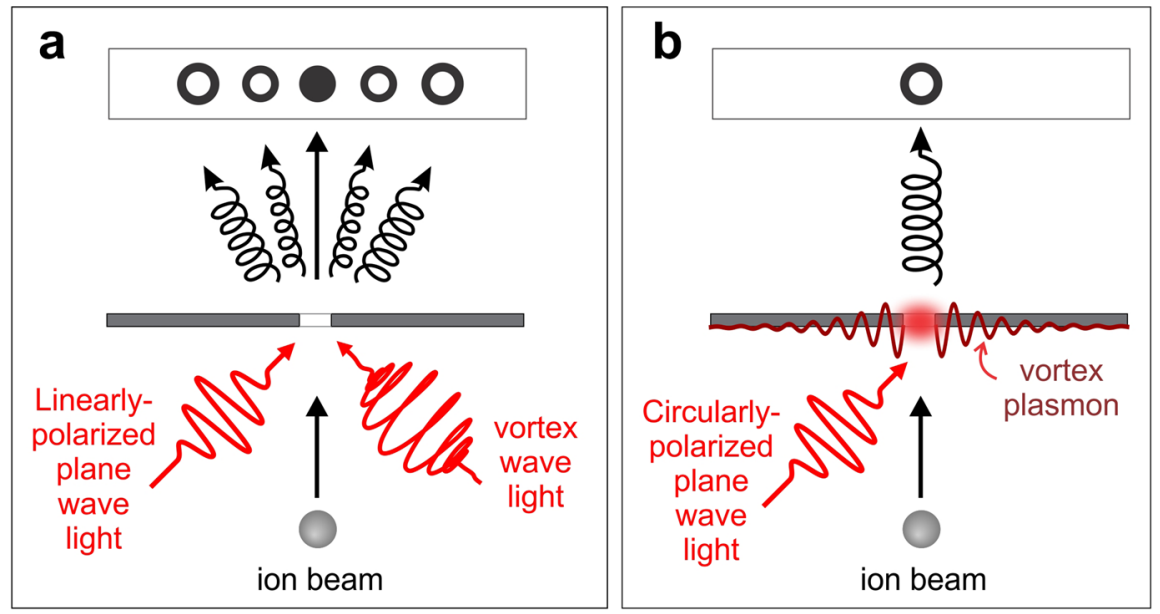

Fig. 12 OAM manipulation of ionized atomic beams. a A stream of ions is diffracting from a light-based fork hologram as made by the coherent superposition of two light beams (one vortex beam and one tilted plane wave). The ponderomotive force governs the atomic diffraction and the generation of a vortex beam in the diffraction orders. b A high-energy ionized atomic beam is interacting with a chiral near-field created at the center of a nano-fabricated hole by illumination with a circularly-polarized light wave. In this case, the full beam carries the same OAM content

able to fiddle with their internal structure and in return their nuclear and electronic energy levels. Until now, interference effects in atom beams have been demonstrated experimentally in various implementations of the double slit experiment [80], and vortex states have been created only with Bose-Einstein condensates [78, 81]. However, no experiment has so far successfully manipulated an ion's wave function and neither it has been prepared in a vortex beam state. Very few theoretical articles considered this possibility so far [82, 83], all highlighting its potential for a broad range of applications, but none realizing the implications for nuclear physics itself. In what follows, we will briefly discuss the difficulties in coherently manipulating ion beams and the potential of such experiments for nuclear physics applications.

Ion beams are typically used for nanofabrication purposes, surface treatment and sputtering [84]. Their transverse and longitudinal manipulation is facilitated by their charge, which makes them sensitive to electromagnetic fields and thus conventional electromagnetic lenses such as solenoids, quadrupoles and RF cavities. Their interaction with a physical object however can be problematic because of electrostatic effects that humper the coherence of the beam and its transmission probability through a phase plate. In intuitive terms, the dangling bonds, or surface states, at the edges of a nanofabricated object trap ions very efficiently resulting in large propagation losses.

In Ref. [33], we have shown the possibility to impart OAM on an electron using a patterned electromagnetic field created by a chiral surface plasmon polariton. The same method would in principle work on any charged particle, including ions. One can imagine to have a stream of ions diffracting from a light-based fork hologram as made by the coherent superposition of two light beams (one vortex beam and one tilted plane wave-see Fig. 12a) [82]. In this case, it is the ponderomotive force 
that governs the atomic diffraction and the generation of a vortex beam. An additional light-based scheme that would work with high-energy ionized atomic beams can be derived from our recent work on ultrafast vortex electrons [33]. In this case, a vortex beam would be generated by the interaction of an ionized beam with a chiral near-field created at the center of a nano-fabricated hole in a metal-dielectric structure sustaining surface plasmon polaritons (see Fig. 12b). In this case the radial distribution of the atomic wave function would still be determined by a Bessel function, and thus the size of the donut obtained in the reciprocal space by observing the beam in the diffraction plane would be given by $\Delta \mathrm{k}_{\text {perp }}=2 \cdot 2 / \mathrm{d}_{\mathrm{h}}$, where $\mathrm{d}_{\mathrm{h}}$ is the hole size. Considering the hole size to be of the order of the light wavelength, the typical donut size would be in the range $5-8 \mu \mathrm{m}^{-1}$. Such a number gives a rough estimate of the feasibility of such experiments, as typical detection sensitivity in modern experiments is indeed in the order of microradiants, comparable to the typical transverse coherence length of atomic beams [80].

In perspective, the use of more coherent atomic beams, such as those obtained by evaporating Bose-Einstein Condensed gases (BEC), often referred to as "atomic lasers" [78, 81], would provide a brighter and more coherent source. A few ideas on the use of such coherent atomic beams for high-energy radiation sources and nuclear physics applications has been recently proposed by us in a perspective article [48]. The general idea is that by patterning the wave function of an atom or an ion via properly designed photonic phase plates $[3,82,83]$ one can influence the inner distribution of masses and charges, thus modifying the matrix elements for the nuclear and electronic transitions and in turn the decay probabilities. In Ref. [48], it was also shown that imparting a singularity at the center of the phase distribution of a nuclear wave function (i.e. creating an atomic vortex beam) results in the modification of the nuclear levels scheme. These modifications can entail energy shifts, but they can also lead to a different symmetry of the excited levels, thus generating new ones while quenching others. We also note that, as obtained in the case of electron pulses where ultrafast light was used to successfully achieve a Rabi-type control of multilevel quantum ladders, the same should be possible for nuclear levels [85] once a switchable handle of the levels population is provided.

\section{Conclusive remarks}

In this review, we have revisited the most recent methods for longitudinal and transverse phase manipulation of a single electron wave function. We developed our discussion within the general theoretical framework of electron-light interaction in presence of either confined polaritons or semi-infinite electromagnetic fields. First, we have explored methods for temporal attosecond modulation of electrons, where the most intense activity is devoted to the experimental realization of a single isolated attosecond electron pulse. Ramsey-type schemes with multiple interacting light pulses have also been discussed in correlation with ultrafast holographic imaging methods for the coherent visualization of low-energy excitations in quantum materials. The recent advancement of longitudinal phase modulation towards the strong coupling regime will be instrumental for electron-photon and electron-electron entanglements in quantum 
applications involving free-electrons. Besides the longitudinal phase modulation, we have also extensively discussed transverse phase shaping by means of localized fields inducing a direct manipulation of either linear momentum or orbital angular momentum of the electron wave packet. We have shown several theoretical and experimental schemes for transverse phase shaping that represent rudimentary precursors of a more complex phase plate for multidimensional and arbitrary electron beam manipulation. The latter would be key in revolutionizing the way materials are investigated in electron microscopy since it would give direct access to specific properties and orders not observable before.

Finally, we have discussed how engineering the wave function of composite particles, such as neutrons, atoms, ions and protons-especially regarding their orbital angular momentum, would give further insights into the fundamental interaction of their inner constituents.

Open Access This article is licensed under a Creative Commons Attribution 4.0 International License, which permits use, sharing, adaptation, distribution and reproduction in any medium or format, as long as you give appropriate credit to the original author(s) and the source, provide a link to the Creative Commons licence, and indicate if changes were made. The images or other third party material in this article are included in the article's Creative Commons licence, unless indicated otherwise in a credit line to the material. If material is not included in the article's Creative Commons licence and your intended use is not permitted by statutory regulation or exceeds the permitted use, you will need to obtain permission directly from the copyright holder. To view a copy of this licence, visit http://creativecommons.org/licenses/by/4.0/.

\section{References}

1. P.G. Merli, G.F. Missiroli, G. Pozzi, Am. J. Phys. 44, 306 (1976)

2. A. Tonomura, J. Endo, T. Matsuda, T. Kawasaki, H. Ezawa, Am. J. Phys. 57, 117 (1989)

3. A.D. Cronin, J. Schmiedmayer, D.E. Pritchard, Rev. Mod. Phys. 81, 1051 (2009)

4. F. Hasselbach, Rep. Prog. Phys. 73, 016101 (2010)

5. C.W. Clark, R. Barankov, M.G. Huber, M. Arif, D.G. Cory, D.A. Pushin, Nature 525, 504 (2015)

6. J. Muller, J.M. Lupton, P.G. Lagoudakis, F. Schindler, R. Koeppe, A.L. Rogach, J. Feldmann, D.V. Talapin, H. Weller, Nano Lett. 5, 2043 (2005)

7. J.C.W. Song, N.M. Gabor, Nat. Nanotechnol. 13, 986 (2018)

8. G. Gibson, J. Courtial, M.J. Padgett, M. Vasnetsov, V. Pas'ko, S.M. Barnett, S. Franke-Arnold, Opt. Express 12, 5448 (2004)

9. N. Bozinovic, Y. Yue, Y. Ren, M. Tur, P. Kristensen, H. Huang, A.E. Willner, S. Ramachandran, Science 340, 1545 (2013)

10. A. Sit, F. Bouchard, R. Fickler, J. Gagnon-Bischoff, H. Larocque, K. Heshami, D. Elser, C. Peuntinger, K. Günthner, B. Heim, C. Marquardt, G. Leuchs, R.W. Boyd, E. Karimi, Optica 4, 1006 (2017)

11. S. Pujals, N. Feiner-Gracia, P. Delcanale, I. Voets, L. Albertazzi, Nat. Rev. Chem. 3, 68 (2019)

12. C. Bevis, R. Karl, J. Reichanadter, D.F. Gardner, C. Porter, E. Shanblatt, M. Tanksalvala, G.F. Mancini, H. Kapteyn, M. Murnane, D. Adams, Ultramicroscopy 184, 164 (2018)

13. D.F. Gardner, M. Tanksalvala, E.R. Shanblatt, X. Zhang, B.R. Galloway, C.L. Porter, R. Karl, C. Bevis, D.E. Adams, H.C. Kapteyn, M.M. Murnane, G.F. Mancini, Nat. Photonics 11, 259 (2017)

14. K.Y. Bliokh, I.P. Ivanov, G. Guzzinati, L. Clark, R. Van Boxem, A. Béché, R. Juchtmans, M.A. Alonso, P. Schattschneider, F. Nori, J. Verbeeck, Phys. Rep. 690, 1 (2017)

15. S.M. Lloyd, M. Babiker, G. Thirunavukkarasu, J. Yuan, Rev. Mod. Phys. 89, 035004 (2017)

16. H. Larocque, I. Kaminer, V. Grillo, G. Leuchs, M.J. Padgett, R.W. Boyd, M. Segev, E. Karimi, Contemp. Phys. 59, 126 (2018)

17. K.E. Priebe, C. Rathje, S.V. Yalunin, T. Hohage, A. Feist, S. Schäfer, C. Ropers, Nat. Photonics 11, 793 (2017)

18. Y. Morimoto, P. Baum, Nat. Phys. 14, 252 (2018) 
19. M. Kozák, T. Eckstein, N. Schönenberger, P. Hommelhoff, Nat. Phys. 14, 121 (2018)

20. G.M. Vanacore, I. Madan, G. Berruto, K. Wang, E. Pomarico, R.J. Lamb, D. McGrouther, I. Kaminer, B. Barwick, F.J. García de Abajo, F. Carbone, Nat. Commun. 9, 2694 (2018)

21. B. Barwick, D.J. Flannigan, A.H. Zewail, Nature 462, 902 (2009)

22. L. Piazza, T.T.A. Lummen, E. Quiñonez, Y. Murooka, B.W. Reed, B. Barwick, F. Carbone, Nat. Commun. 6, 6407 (2015)

23. E. Pomarico, I. Madan, G. Berruto, G.M. Vanacore, K. Wang, I. Kaminer, F.J. García de Abajo, F. Carbone, ACS Photonics 5, 759 (2018)

24. G.M. Vanacore, A.W.P. Fitzpatrick, A.H. Zewail, Nano Today 11, 228 (2016)

25. A. Yurtsever, R.M. van der Veen, A.H. Zewail, Science 335, 59 (2012)

26. D.L. Freimund, K. Aflatooni, H. Batelaan, Nature 413, 142 (2001)

27. S.T. Park, M. Lin, A.H. Zewail, New J. Phys. 12, 123028 (2010)

28. F.J. Garcia De Abajo, A. Asenjo-Garcia, M. Kociak, Nano Lett. 10, 1859 (2010)

29. A. Feist, K.E. Echternkamp, J. Schauss, S.V. Yalunin, S. Schäfer, C. Ropers, Nature 521, 200 (2015)

30. D.J. Flannigan, B. Barwick, A.H. Zewail, Proc. Natl. Acad. Sci. USA 107, 9933 (2010)

31. I. Madan, G.M. Vanacore, E. Pomarico, G. Berruto, R.J. Lamb, D. McGrouther, T.T.A. Lummen, T. Latychevskaia, F.J. García de Abajo, F. Carbone, Sci. Adv. 5, eaav8358 (2019)

32. K.E. Echternkamp, A. Feist, S. Schäfer, C. Ropers, Nat. Phys. 12, 1000 (2016)

33. G.M. Vanacore, G. Berruto, I. Madan, E. Pomarico, P. Biagioni, R.J. Lamb, D. McGrouther, O. Reinhardt, I. Kaminer, B. Barwick, H. Larocque, V. Grillo, E. Karimi, F.J. García de Abajo, F. Carbone, Nat. Mater. 18, 573 (2019)

34. C. Kealhofer, W. Schneider, D. Ehberger, A. Ryabov, F. Krausz, P. Baum, Science 352, 429 (2016)

35. A. Gover, E. Dyunin, IEEE J. Quantum Electron. 46, 1511 (2010)

36. Y. Pan, B. Zhang, A. Gover, Phys. Rev. Lett. 122, 183204 (2019)

37. O. Reinhardt, I. Kaminer, ACS Photonics 7, 2859 (2020)

38. O. Kfir, H. Lourenço-Martins, G. Storeck, M. Sivis, T.R. Harvey, T.J. Kippenberg, A. Feist, C. Ropers, Nature 582, 46 (2020)

39. K. Wang, R. Dahan, M. Shentcis, Y. Kauffmann, A.B. Hayun, O. Reinhardt, S. Tsesses, I. Kaminer, Nature 582, 50 (2020)

40. M. Kozák, J. McNeur, K.J. Leedle, H. Deng, N. Schönenberger, A. Ruehl, I. Hartl, J.S. Harris, R.L. Byer, P. Hommelhoff, Nat. Commun. 8, 14342 (2017)

41. Y. Morimoto, P. Baum, Phys. Rev. A 97, 033815 (2018)

42. P. Baum, F. Krausz, Chem. Phys. Lett. 683, 57 (2017)

43. M. Kozák, Phys. Rev. Lett. 123, 203202 (2019)

44. M.T. Hassan, J.S. Baskin, B. Liao, A.H. Zewail, Nat. Photonics 11, 425 (2017)

45. Y. Morimoto, P. Baum, Phys. Rev. Lett. 125, 193202 (2020)

46. M.T. Hassan, H. Liu, J.S. Baskin, A.H. Zewail, Proc. Nat. Acad. Sci. USA 112, 12944 (2015)

47. X. Fu, F. Barantani, S. Gargiulo, I. Madan, G. Berruto, T. Lagrange, L. Jin, J. Wu, G.M. Vanacore, F. Carbone, Y. Zhu, Nat. Commun. 11, 5770 (2020)

48. I. Madan, G.M. Vanacore, S. Gargiulo, T. LaGrange, F. Carbone, Appl. Phys. Lett. 116, 230502 (2020)

49. A. Asenjo-Garcia, F.J. García de Abajo, New J. Phys. 15, 103021 (2013)

50. O. Kfir, Phys. Rev. Lett. 123, 103602 (2019)

51. V. Di Giulio, M. Kociak, F.J.G. de Abajo, Optica 6, 1524 (2019)

52. Y. Pan, A. Gover, Phys. Rev. A 99, 052107 (2019)

53. S. Tsesses, G. Bartal, I. Kaminer, Phys. Rev. A 95, 013832 (2017)

54. R. Remez, A. Karnieli, S. Trajtenberg-Mills, N. Shapira, I. Kaminer, Y. Lereah, A. Arie, Phys. Rev. Lett. 123, 60401 (2019)

55. G. Guzzinati, A. Béché, H. Lourenço-Martins, J. Martin, M. Kociak, J. Verbeeck, Nat. Commun. 8, (2017).

56. D. Ehberger, K.J. Mohler, T. Vasileiadis, R. Ernstorfer, L. Waldecker, P. Baum, Phys. Rev. Appl. 11, 024034 (2019)

57. A. Feist, S.V. Yalunin, S. Schafer, C. Ropers, Phys. Rev. Res. 2, 043227 (2020)

58. R. Kienberger, E. Goulielmakis, M. Uiberacker, A. Baltuska, V. Yakovlev, F. Bammer, A. Scrinzi, T. Westerwalbesloh, U. Kleineberg, U. Heinzmann, M. Drescher, F. Krausz, Nature 427, 817 (2004)

59. D.L. Freimund, H. Batelaan, Phys. Rev. Lett. 89, 283602 (2002)

60. F.J. García de Abajo, B. Barwick, F. Carbone, Phys. Rev. B 94, 041404 (2016)

61. N. Talebi, C. Lienau, New J. Phys. 21, 093016 (2019) 
62. J.T. Collins, C. Kuppe, D.C. Hooper, C. Sibilia, M. Centini, V.K. Valev, Adv. Opt. Mater. 5, 1700182 (2017)

63. A. Asenjo-Garcia, F.J. García de Abajo, Phys. Rev. Lett. 113, 066102 (2014)

64. R. Juchtmans, A. Béché, A. Abakumov, M. Batuk, J. Verbeeck, Phys. Rev. B 91, 094112 (2015)

65. M. Uchida, A. Tonomura, Nature 464, 737 (2010)

66. J. Verbeeck, H. Tian, P. Schattschneider, Nature 467, 301 (2010)

67. B.J. McMorran, A. Agrawal, I.M. Anderson, A.A. Herzing, H.J. Lezec, J.J. McClelland, J. Unguris, Science 331, 192 (2011)

68. E. Mafakheri, A.H. Tavabi, P.-H. Lu, R. Balboni, F. Venturi, C. Menozzi, G.C. Gazzadi, S. Frabboni, A. Sit, R.E. Dunin-Borkowski, E. Karimi, V. Grillo, Appl. Phys. Lett. 110, 093113 (2017)

69. A. Béché, R. Van Boxem, G. Van Tendeloo, J. Verbeeck, Nat. Phys. 10, 26 (2013)

70. J. Verbeeck, A. Béché, K. Müller-Caspary, G. Guzzinati, M.A. Luong, M. Den Hertog, Ultramicroscopy 190, 58 (2018)

71. G. Pozzi, P.H. Lu, A.H. Tavabi, M. Duchamp, R.E. Dunin-Borkowski, Ultramicroscopy 181, 1339 (2017)

72. J. Handali, P. Shakya, B. Barwick, Opt. Express 23, 5236 (2015)

73. W. Cai, O. Reinhardt, I. Kaminer, F.J.G. De Abajo, Phys. Rev. B 98, 45424 (2018)

74. G. Spektor, D. Kilbane, A.K. Mahro, B. Frank, S. Ristok, L. Gal, P. Kahl, D. Podbiel, S. Mathias, H. Giessen, F.J.M. Zu Heringdorf, M. Orenstein, M. Aeschlimann, Science 355, 1187 (2017)

75. K.Y. Bliokh, F.J. Rodríguez-Fortuño, F. Nori, A.V. Zayats, Nat. Photonics 9, 796 (2015)

76. A. Konečná, F.J.G. de Abajo, Phys. Rev. Lett. 125, 030801 (2020)

77. O. Schwartz, J.J. Axelrod, S.L. Campbell, C. Turnbaugh, R.M. Glaeser, H. Müller, Nat. Methods 16, 1016 (2019)

78. M.R. Matthews, B.P. Anderson, P.C. Haljan, D.S. Hall, C.E. Wieman, E.A. Cornell, Phys. Rev. Lett. 83, 2498 (1999)

79. H. Larocque, I. Kaminer, V. Grillo, R.W. Boyd, E. Karimi, Nat. Phys. 14, 1 (2018)

80. O. Carnal, J. Mlynek, Phys. Rev. Lett. 66, 2689 (1991)

81. M. Andersen, C. Ryu, P. Cladé, V. Natarajan, A. Vaziri, K. Helmerson, W. Phillips, Phys. Rev. Lett. 97, 170406 (2006)

82. V.E. Lembessis, D. Ellinas, M. Babiker, O. Al-Dossary, Phys. Rev. A 89, 053616 (2014)

83. V.E. Lembessis, Phys. Rev. A 96, 013622 (2017)

84. F. Watt, A.A. Bettiol, J.A. Van Kan, E.J. Teo, M.B.H. Breese, Int. J. Nanosci. 4, 269 (2005)

85. J. Haber, X. Kong, C. Strohm, S. Willing, J. Gollwitzer, L. Bocklage, R. Rüffer, A. Pálffy, R. Röhlsberger, Nature Photonics 11, 720-725 (2017)

\section{Affiliations}

\section{Giovanni Maria Vanacore ${ }^{1} \cdot$ Ivan Madan $^{2} \cdot$ Fabrizio Carbone $^{2}$}

$凶 \quad$ Fabrizio Carbone

fabrizio.carbone@epfl.ch

1 Laboratory of Ultrafast Microscopy for Nanoscale Dynamics (LUMiNaD), Department of Materials Science, University of Milano-Bicocca, Via Cozzi 55, 20125 Milan, Italy

2 Laboratory for Ultrafast Microscopy and Electron Scattering (LUMES), Institute of Physics (IPHYS), École Polytechnique Fédérale de Lausanne (EPFL), Lausanne, Switzerland 